\title{
HISTORISCHE PROBLEME DER 3. ZWISCHENZEIT ${ }^{1}$
}

\author{
Von KARL JANSEN-WINKELN
}

\begin{abstract}
Republication of a statue of Bes in the Oriental Museum, Durham, showing that interpretations based on earlier copies are untenable. The statue was dedicated by a High Priest Shoshenq, but his son Harsiese was only an ordinary prophet of Amun. Starting from the chronology of Aston, $\mathcal{Y} E A$ 75, the author distinguishes the king, Harsiese A, from a High Priest, Harsiese B, who held office under Osorkon II. The slightly earlier High Priest Takeloth $\mathrm{F}$ is identified with the later king Takeloth II. It is further suggested that the 'Theban Twenty-third Dynasty' consists of two rival lines, each with its own High Priest: 1) Takeloth II and Iuput I, with High Priest Osorkon B (perhaps resident at Herakleopolis), and 2) Pedubast I and Shoshenq IV, with High Priests Harsiese B followed by Takeloth E (perhaps resident at Hermopolis). Since Pedubast is also attested in Lower Egypt, he probably had allies there. Finally, the High Priest Shoshenq of the Bes statue is identified with the king Shoshenq who renewed the statue Cairo CG 42192 for Psusennes II.
\end{abstract}

\section{Harsiese A}

ÜBER den König Harsiese A, der im 9. Jahrhundert in Theben herrschte und dort auch begraben ist, scheint zur Zeit folgendes sicher bekannt zu sein: ${ }^{2}$

-Er amtierte während der Regierungszeit Osorkons II.; ${ }^{3}$

-er war an sich Hoherpriester des Amun, der sich dann den Königstitel anmaßte, wie das ja in dieser Zeit (und v.a. in der 21. Dynastie) auch sonst vorkommt; ${ }^{4}$

-er war der Sohn des Hohenpriesters Schoschenk (II.), des Sohnes Osorkons I., und diese Abstammung ist zugleich als ein Grund dafür angesehen worden, warum sich Harsiese die Königstitulatur zulegte: Schon sein Vater umgibt gelegentlich seinen Namen mit der Kartusche, und er ist nach verbreiteter Ansicht auch mit dem in Tanis bestatteten König $H q_{3}-h p r-R r$ identisch. ${ }^{5}$

Für diese genealogische Verbindung, die in der gesamten Literatur unumstritten ist, ${ }^{6}$ beruft man sich auf eine Statue des Gottes Bes im Oriental Museum der University of Durham (ehemals Sammlung Alnwick Castle), die der Hohepriester Schoschenk zusammen mit seinem Sohn Harsiese gestiftet hat. Die Inschriften darauf sind von S. Birch

\footnotetext{
${ }^{1}$ Die zur Unterscheidung der Personen verwendeten Kennziffern und -buchstaben richten sich nach K. A. Kitchen, The Third Intermediate Period in Egypt ${ }^{2}$ (Warminster 1986; im folgenden TIP ${ }^{2}$ ) und M. L. Bierbrier, The Late New Kingdom in Egypt (Warminster 1975). Soweit nicht anders erwähnt, Abkürzungen nach Lexikon der Ägyptologie, VII, xiv-xxxviii.

${ }^{2} \mathrm{Vgl}$. zusammenfassend $T I P^{2}, \S 272-5$.

${ }^{3}$ Auf der Statue Kairo CG 42208 ist die ausführliche Titulatur Osorkons II. auf dem Pantherfell der dargestellten Person zu lesen; als 'Stifter' der Statue (in der $d j-m-h z w t$-Formel) erscheint aber Harsiese A, s. meine Ägyptischen Biographien der 22. und 23. Dynastie (ÄUAT 8, Wiesbaden 1985; im folgenden Äg. Biogr.), 453. Auf der Statue Kairo CG 42225 ist der 'Stiftungsvermerk' eines Hohenpriesters Harsiese auf der Schulter neben der Kartusche Osorkons II. angebracht, s. ibid., 494.

${ }^{4} \mathrm{~S} . T I P^{2}, \S 274$.

${ }^{5} \mathrm{Vgl}$. TIP 2 , §§ 93-4; 269; 452.

${ }^{6}$ Z. B. LR III, 331, E (mit n. 2); 348, I (mit n. 2); E. Meyer, Gottesstaat, Militärherrschaft und Ständewesen in Agypten (SPAW, Berlin 1928), 514; H. Kees, Die Hohenpriester des Amun von Karnak von Herihor bis zum Ende der Äthiopenzeit (Leiden 1964), 92-3; 109; W. Helck, 'Harsiese', LÄ II, 1020; TIP2, § 265; 272, n. 395; J. von Beckerath, Handbuch der ägyptischen Königsnamen (MÄS 20, München u. Berlin 1984), 104, n. 10 u.a.m.
} 
im Catalogue of the Egyptian Antiquities at Alnwick Castle (London 1880), 33-4 ziemlich fehlerhaft publiziert worden. Sie werden aber fast nie nach dieser Publikation zitiert, sondern nach einer Auflistung der Namen und Titel darin durch G. Legrain in RecTrav 30 (1908), 160. Da die Angaben Legrains stark von denen bei Birch abweichen, hat man sich offenbar darauf verlassen, daß er den Text kollationiert hatte, obwohl er nichts dergleichen sagt. Tatsächlich sind aber gerade die Aufstellungen Legrains vollkommen irreführend: In seinem 'tableau généalogique' hat er die Angaben verschiedener Texte (der Besstatue, der Nilstatue London BM 8, der Papyri Denon B und C, s.u.) kombiniert, bringt sie aber unter der Überschrift 'Statue de Bès de Alnwick Castle'. Der Sohn des Hohenpriesters Schoschenk namens Osorkon und seine Mutter Ns-t;-wdit-3ht sind zusammen nur auf dem pDenon $\mathrm{C}$ bezeugt, und die besondere Titulatur Schoschenks (einschließlich Kartuschennamen), die Legrain anführt, stammt von der Nilstatue, nicht von der Besstatue!

Die Verwirrung ist komplett geworden, als Gauthier die Angaben Legrains ungeprüft übernommen hat und in seinem Livre des rois ${ }^{7}$ jetzt für die Besstatue den Text gibt, der in Wirklichkeit von der Nilstatue BM 8 stammt. Von Legrain und Gauthier aus ist dann dieser Irrtum in die gesamte übrige Literatur eingegangen. Auch ich selbst bin ihm zum Opfer gefallen, s. GM 99 (1987), 19, wo der Beleg Nr. 8 zu streichen ist (=Nr. 7). ${ }^{8}$ Tatsächlich kommen auf der Besstatue nur der Hohepriester Schoschenk, seine Frau $N s-n b(t)-\mathscr{F} r z w$ und deren gemeinsamer Sohn Harsiese vor, und der ist zudem kein Hoherpriester, sondern einfacher Amunprophet. ${ }^{9}$

Die folgende Neuveröffentlichung der Inschriften der Besstatue aus Durham (s. Pl. XI-XII) basiert auf Fotos, die mir John Ruffle vom Oriental Museum Durham freundlicherweise zur Verfügung stellte, der auch meine nach den Fotos angefertigte Zeichnung noch einmal kollationiert hat, wofür ich ihm herzlich danke.

\section{Inschrift auf der Oberseite des Sockels (Fig. 1)}

Gemacht vom Ersten Propheten des Amonrasonther Schoschenk, gerechtfertigt, ${ }^{a}$ dem Sohn des Königs, des Herrn der Beiden Länder Mrjj-尹̈mn Osorkon, und seinem Sohn

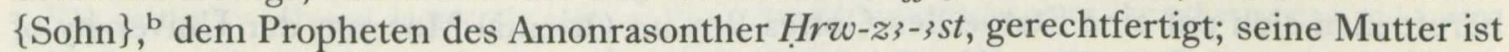
die Sängerin vom Inneren der Obersten der Beiden $\operatorname{Länder}^{c} N s-n b(t)$ - $\mathscr{s}$ šrw, gerechtfertigt, für seine ${ }^{d}$ Herrin Isis zu Gast in Behbeit, die Leben, Heil und Gesundheit gibt unaufhörlich[??].

\section{Inschrift um den Sockel (Fig. 2)}

[Gemacht von] dem Ersten Propheten des Amonrasonther, dem Generalissimus und Befehlshaber Schoschenk, gerechtfertigt, dem Sohn des Königs, des Herrn der Beiden Länder Mrjj-Imn Osorkon, und seinem Sohn, dem Propheten des Amonrasonther Hrwz3-3st, gerechtfertigt; seine Mutter ist die Sängerin vom Inneren der Obersten der Beiden

${ }^{7}$ LR III, 331, E (mit n. 2).

${ }^{8}$ Die scheinbaren Lesungsunterschiede ergeben sich daraus, daß ich die Nilstatue nach J. Leibovitch, 'Gods of Agriculture and Welfare in Ancient Egypt', FNES 12 (1953), 80 zitiert hatte, während Legrain denselben Text nach Lepsius, Auswahl, Taf. xv wiedergibt.

${ }^{9}$ Übrigens hatte schon G. Daressy in 'Notes sur les XXII', XXIII ${ }^{\mathrm{e}}$ et XXIV ${ }^{\mathrm{e}}$ dynasties', RecTrav 35 (1913), 143 n. 2 auf den Irrtum Legrains hingewiesen (obgleich seine Feststellungen ihrerseits nicht völlig korrekt sind), aber das scheint nirgends beachtet worden zu sein. 


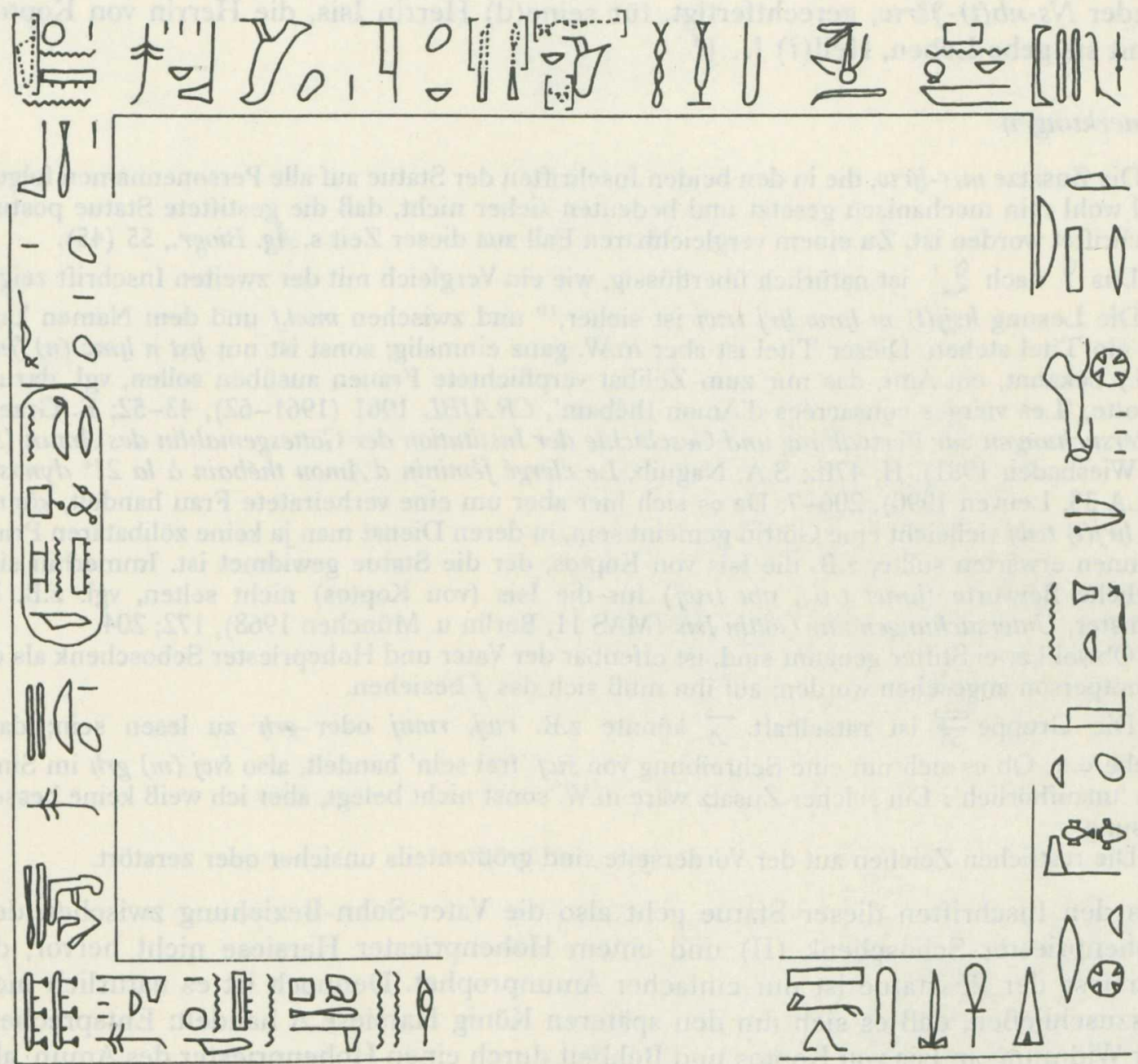

Fig. 1. Besstatue Durham: Inschrift auf der Oberseite des Sockels.

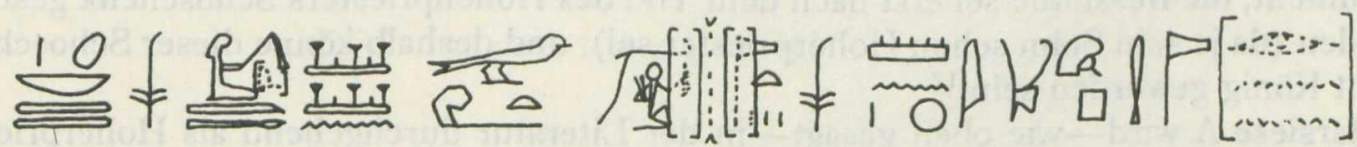

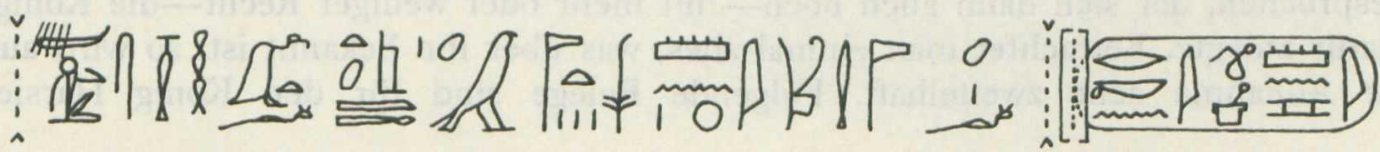

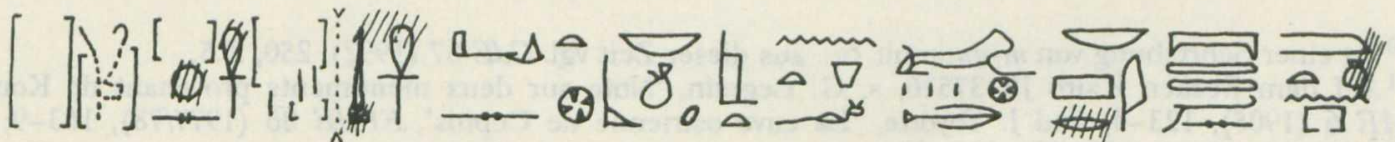

Fig. 2. Besstatue Durham: Inschrift um den Sockel. 
Länder $N s-n b(t)-\mathcal{Y s ̌ r w}$, gerechtfertigt, für seine(d) Herrin Isis, die Herrin von Koptos, damit sie gebe Leben, Heil(?) [...]. ${ }^{\mathrm{f}}$

\section{Anmerkungen}

${ }^{a}$ Die Zusätze $m$; $r-h r w$, die in den beiden Inschriften der Statue auf alle Personennamen folgen, sind wohl rein mechanisch gesetzt und bedeuten sicher nicht, daß die gestiftete Statue postum beschriftet worden ist. Zu einem vergleichbaren Fall aus dieser Zeit s. Ag. Biogr., 55 (45).

${ }^{\mathrm{b}}$ Das ${ }^{\prime}$ nach ${ }^{0} 1$ ist natürlich überflüssig, wie ein Vergleich mit der zweiten Inschrift zeigt.

${ }^{\mathrm{C}}$ Die Lesung $h s j j(t) m$ hnww hrj $t_{3} w j$ ist sicher, ${ }^{10}$ und zwischen mwt.f und dem Namen kann nur ein Titel stehen. Dieser Titel ist aber m.W. ganz einmalig; sonst ist nur hst $n$ hnw (n) $7 m n$ (u.ä.) bekannt, ein Amt, das nur zum Zölibat verpflichtete Frauen ausüben sollen, vgl. dazu J. Yoyotte, 'Les vierges consacrées d'Amon thébain', CRAIBL 1961 (1961-62), 43-52; E. Graefe, Untersuchungen zur Verwaltung und Geschichte der Institution der Gottesgemahlin des Amun (ÄA 37, Wiesbaden 1981). II, 47ff.; S.A. Naguib, Le clergé féminin d'Amon thébain à la $21^{\mathrm{e}}$ dynastie (OLA 38, Leuven 1990), 206-7. Da es sich hier aber um eine verheiratete Frau handelt, könnte mit $h r j(t) t$ tswj vielleicht eine Göttin gemeint sein, in deren Dienst man ja keine zölibatären Priesterinnen erwarten sollte, z.B. die Isis von Koptos, der die Statue gewidmet ist. Immerhin sind ähnliche Beiworte (hnwt tzwj, nbt tzwj) für die Isis (von Koptos) nicht selten, vgl. z.B. M. Münster, Untersuchungen zur Göttin Isis (MÄS 11, Berlin u. München 1968), 172; 204.

${ }^{\mathrm{d}}$ Obwohl zwei Stifter genannt sind, ist offenbar der Vater und Hohepriester Schoschenk als die Hauptperson angesehen worden; auf ihn muß sich das $f$ beziehen.

${ }^{e}$ Die Gruppe $\vec{\Delta}$ ist rätselhaft. $\vec{\Delta}$ könnte z.B. $r w j, r m n j$ oder grh zu lesen sein; dann bliebe $\square$. Ob es sich um eine Schreibung von šzuj 'frei sein' handelt, also šzuj (m) grh im Sinne von 'unaufhörlich'? Ein solcher Zusatz wäre m.W. sonst nicht belegt, aber ich weiß keine bessere Lösung.

${ }^{\mathrm{f}}$ Die restlichen Zeichen auf der Vorderseite sind größtenteils unsicher oder zerstört.

Aus den Inschriften dieser Statue geht also die Vater-Sohn-Beziehung zwischen dem Hohenpriester Schoschenk (II) und einem Hohenpriester Harsiese nicht hervor, der Harsiese der Besstatue ist nur einfacher Amunprophet. Dennoch ist es natürlich nicht auszuschließen, daß es sich um den späteren König Harsiese A handelt: Entsprechend der Widmung an Isis von Koptos und Behbeit durch einen Hohenpriester des Amun, also den Machthaber Oberägyptens, dürfte die Statue aus Koptos stammen, und Harsiese A ist (mit seinem Sohn) ja auch in Koptos bezeugt. ${ }^{11}$ Die bisher angenommene Genealogie muß also nicht notwendig falsch sein, sie wird aber durch die Besstatue nicht erwiesen. Vorläufig muß die Abstammung des Harsiese A offenbleiben. Des weiteren entfällt das Argument, die Besstatue sei erst nach dem Tod des Hohenpriesters Schoschenk gestiftet worden (da ja sein Sohn schon Hoherpriester sei), und deshalb könne dieser Schoschenk nicht König geworden sein. ${ }^{12}$

Harsiese A wird-wie oben gesagt-in der Literatur durchgehend als Hoherpriester angesprochen, der sich dann auch noch-mit mehr oder weniger Recht-die Königstitulatur zulegte. Betrachtet man einmal alles, was über ihn bekannt ist, so wird auch diese Annahme sehr zweifelhaft. Folgende Belege sind für den König Harsiese

${ }^{10} \mathrm{Zu}$ einer Schreibung von $m$-hnnw mit o॰ aus dieser Zeit vgl. CdE 67 (1992), 250, Z.5.

${ }^{11}$ Auf dem Becken Kairo JE 37516, s. G. Legrain, 'Note sur deux monuments provenant de Kouft', ASAE 6 (1905), 123-4, und J. Yoyotte, 'La cuve osirienne de Coptos', EPHE 86 (1977/78), 163-9; 88 (1979/80), 194-7; 90 (1981/82), 189-92.

${ }^{12}$ So H. Jacquet-Gordon, BiOr 32 (1975), 359; vgl. auch I. E. S. Edwards, $C A H^{2}$, III/1 (1982), 551. 
vorhanden: ${ }^{13}$

A. Becken aus Koptos ${ }^{14}$

König Harsiese opfernd vor Osiris; Beischrift:

Hrw: $K_{3}-n h t$ H ${ }^{j} j-m-W_{3} s t$ [im Serech]

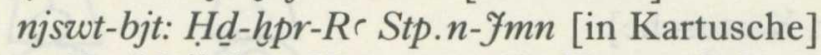

$z_{3} R r: M r j j-9 m n$ Hrw-z3-3st [in Kartusche]

Auf der gegenüberliegenden Seite räuchernd und libierend sein Sohn ( $z_{3}$ njswt nb $t_{3} w j$ Mrij- Imn Hrw-z3-3st [in Kartusche]), ein (Hoher?)Priester des Amun, dessen Namen zum großen Teil zerstört ist. ${ }^{15}$

B. Sarkophag Kairo JE 59896 aus seinem Grab in Medinet $\mathrm{Habu}^{16}$ Auf dem Deckel die Titulatur:

Hrw: K3-nht Hij-m-W $W_{3}$ st [im Serech] njswt-bjt: Hd-hpr-Re Stp. $n$ - Imn [in Kartusche] z3 Re: Mrjj- Imn Hrw-z;-3st [in Kartusche]

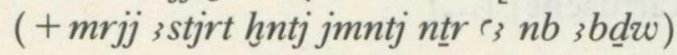

C. 4 Kanopen Kairo JE 59900a-d aus demselben Grab ${ }^{17}$

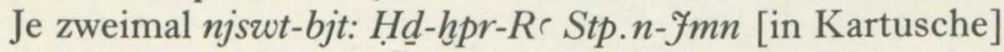
und $z$-R : Mrjj- $7 m n$ Hrw-z;-3st [in Kartusche]

D. Stele Berlin $14995^{18}$

König Harsiese opfernd vor Amon-Re-Kamutef, hinter ihm die Gottesgemahlin $\mathrm{Mrjj}$ Mwt 'Karomama'; Beischrift:

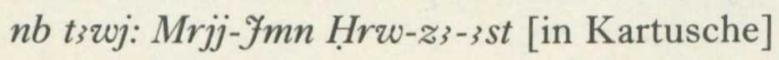

E. 'Les restes d'une petite porte en grès' am rechten Flügel des IV. Pylons in Karnak ${ }^{19}$ König Harsiese mit oberägyptischer Krone; Beischrift:

njswt-bjt: Hd-hpr-Re Stp.n-Imn [in Kartusche] z3 Rr: Mrjj-7mn Hrw-zs-3st [in Kartusche]

F. Oberhalb der Säulen 18 und 19 im Vorhof des Chonstempels von Karnak ${ }^{20}$ 'Cartouches of Harsiesi' [wohl wie in E]

G. Stelophor Kairo CG $42208^{21}$

Auf dem oberen Rand der Stele Stiftungsformel dj(w) $m$ ḩzwt nt hr njswt nb tiwj: Mrjj-

${ }^{13}$ Der König Hrw-zz-3st [in Kartusche], Sohn des Osiris Dd-Hrw [in Kartusche] auf der Statue Brüssel E. 7654 (s. M.-A. Bonhême, Les noms royaux dans l'Egypte de la Troisième Période Intermédiaire (BdE 98, Kairo 1987), 195 n. 3) gehört sicher nicht hierher.

${ }^{14}$ S.o., Anm. 11.

${ }^{15} \mathrm{Zu}$ dieser Person, s.u., p. 000.

${ }^{16}$ S. U. Hölscher, The Excavation of Medinet Habu, V. Post-Ramessid Remains (Chicago 1954), 10; pl. 8 B; $10 \mathrm{D}$.

${ }^{17}$ Ibid., 10; pl. 10 C.

${ }^{18} \mathrm{Berl}$. Inschr., II, 210.

${ }^{19} \mathrm{P}$. Barguet, Le temple d'Amon-Rê à Karnak (Kairo 1962), 92; $\mathrm{PM} \mathrm{II}^{2}, 78$.

${ }^{20}$ Unpubliziert, vgl. $\mathrm{PM} \mathrm{II}^{2}, 232$.

${ }^{21}$ Äg. Biogr., 453, b, 1-2. 


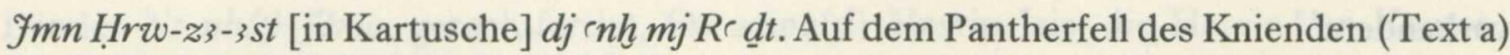
die ausführliche Titulatur Osorkons II.

H. Würfelhocker Kairo CG $42254=\mathrm{JE} 37374^{22}$

Auf den Schultern (Fig. 3):

rechts: Hd-hpr-Re Stp. $n-R e$ (sic) [in Kartusche]

links: Mrjj-Jmn Hrw-z3-3st [in Kartusche]

I. Würfelhocker Kairo CG $42210^{23}$

Auf den Schultern: In der Stiftungsformel wird die Mutter des Stifters (und Frau des Dargestellten) 3stwrt bezeichnet als

zst njswt nb tzwj Mrjj-Imn Hrw-z3-3st [in Kartusche]

J. Würfelhocker Kairo CG $42211^{24}$

Auf der Rückseite dieselbe 3 stwrt, die Mutter des Statuenbesitzers, als

zst njswt nb t3wj Mrjj- Fmn Hrw-z;-3st [in Kartusche] [...]

K. Nr. 23 der 'Priesterannalen' aus Karnak ${ }^{25}$

Offenbar dieselbe 3 stwrt ist

zst njswt nb t;wj Mrjj--7mn [Hrw-z3]-3st [in Kartusche] enh $\underline{d} t$

L. Auf den Kartonagen ihrer Töchter T3-hnmt und Dd-Mwt-jw.s- $n h^{26}$ ist 3stwrt zst njswt nb t;wj Mrjj-Fmn Hrw-z;-3st [in Kartusche] dj enh (mj Re [...])

M. Sarg(?)fragment Philadelphia, Pennsylvania University Museum E. 16186 aus Abydos $^{27}$

Eine $\left[T_{3}-d j-t\right] 3-n b(t)-h n m_{3} c_{-}-h r w$ ist zst njswt nb tзwj Mrjj- Fmn Hrw-z;-3st [in Kartusche]

N. Statuette Cambridge, Fitzwilliam Museum E. 11.1937 aus der 25.-26. Dynastie ${ }^{28}$ Der Besitzer $W n$ - $n f r$ ist wih-mw njswt-bjt Hrw-z;-3st [in Kartusche]

O. Schreiberstatue Kairo JE 37398 aus dem Beginn der 26. Dynastie ${ }^{29}$ Der Besitzer $P_{3}$-dj-手mn-nb-nswt-t;wj ist u.a.

hm-ntr $n$ t3 hivt $n$ njswt Hrw-z3-3st [in Kartusche] m3r-hrw.

${ }^{22}$ Unpubliziert, nach eigener Abschrift, s. Fig. 3. Vgl. ibid., 263.

${ }^{23}$ Ibid., 462.

${ }^{24}$ Ibid., 475, k, 8.

${ }^{25}$ Legrain, 'Notes prises à Karnak', RecTrav 22 (1900), 59; J.-M. Kruchten, Les annales des prêtres de Karnak (OLA 32, Löwen 1989), 115, pl. 18.

${ }^{26}$ J. E. Quibell u. W. Spiegelberg, The Ramesseum (London 1898), pl. xxiv, 4 bzw. xxv, 3.

${ }^{27} \mathrm{~J}$. Taylor, 'A Daughter of King Harsiese', $\mathscr{F E A} 74$ (1988), 230-1.

${ }^{28} \mathrm{Ch}$. Insley, 'A Bronze Statue of Unnufer, Choachyte of King Harsiese, in the Fitzwilliam Museum', $\mathscr{F} E A$ 65 (1979), 167-9; pl. xxx-xxxi.

${ }^{29}$ G. Vittmann, Priester und Beamte im Theben der Spätzeit (Veröffentlichungen der Institute für Afrikanistik und Âgyptologie der Universität Wien, 3, Wien 1978), 72-4, Taf. 1/2. 
Der König Harsiese ist also $15 \mathrm{x}$ belegt: ${ }^{30}$

-Als 'Besitzer' (o.ä.) in den Belegen A $(3,4,5)$; B $(3,4,5)$; C (2x 4; 2x 5); D (5);

-als 'Bauherr/Restaurator' (o.ä.) in $\mathbf{E}(4,5)$ und $\mathbf{F}(4,5$ [?]);

-als 'Stifter' (o.ä., von Privatstatuen) in $\mathbf{G}(5)$ und $\mathbf{H}(4,5)$;

-in der Genealogie seiner Töchter $3 s t w r t$ in I-L (jeweils 5) bzw. $[T ; d j-t] 3-n b(t)-h n$ in $\mathbf{M}(5)$;

-im späteren Kult in $\mathbf{N}(5)$ und $\mathbf{O}$ (5).

Der Beleg $\mathbf{A}$ ist aus Koptos, $\mathbf{M}$ aus Abydos, ${ }^{31}$ alle anderen sind aus Theben.

\section{Harsiese B}

Dem Hohenpriester Harsiese (A) wurden bislang zwei Belege zugeordnet: die Besstatue von Durham sowie Kairo CG 42225. Da, wie gesehen, auf der Besstatue gar kein Hoherpriester Harsiese erwähnt wird, bleibt nur noch ein einziger Fall übrig: Auf dem Würfelhocker Kairo CG $42225^{32}$ stehen auf den Schultern die Kartuschen Osorkons II. sowie, auf der rechten Schulter, neben dem Eigennamen Osorkons, ein zusätzlicher

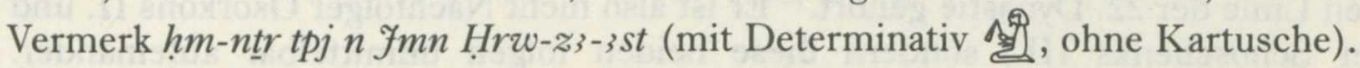

Allein die Tatsache, daß einem einzigen Beleg für den Hohenpriester Harsiese relativ zahlreiche für den König gegenüberstehen, wäre auffällig. Dazu kommt noch Folgendes: Die Statue, um die es geht, gehört Nebneteru III. und ist nach seinem Tod von seinem Enkel Hor IX aufgestellt worden. ${ }^{33}$ Dieser Enkel war seinerseits mit einer Enkelin Osorkons II. verheiratet und ein Zeitgenosse der Könige Petubastis und Schoschenk III. ${ }^{34}$ Das spricht doch sehr dafür, daß die Statue Kairo CG 42225 ganz ans Ende der Regierungszeit Osorkons II. gehört. ${ }^{35}$ Harsiese A sollte dagegen eher zu Beginn der langen Regierungszeit Osorkons II. amtiert haben: Auf einer Statue, die die Kartuschen Osorkons II. trägt, steht eine Fürbitte für einen Urenkel des Nachtefmut A. ${ }^{36}$ Dieser Nachtefmut A gehört zur selben Generation wie Harsiese A (sein Sohn ist mit einer Tochter des Harsiese verheiratet), und seine eigene Statue (Kairo CG 42208, s.o., G) hat schon einen Vermerk des Königs Harsiese. Es wäre schon mehr als merkwürdig, wenn nun Harsiese A am Ende der Amtszeit Osorkons II. als Hoherpriester auftauchte, nachdem er lange vorher schon als König bezeugt war.

\footnotetext{
${ }^{30}$ Die Ziffern hinter den Belegen geben die jeweils verwendeten Titel wieder: $3=$ Horusname im Serech; $4=n j s w t-b j t-\mathrm{Name}$ in Kartusche; $5=z_{3}-R r-\mathrm{Name}$ in Kartusche.

${ }^{31}$ Es ist natürlich nicht gesagt, daß der Sarg der Tochter des Harsiese selbst gehört und diese Tochter mithin in Abydos belegt wäre. Im erhaltenen Fragment könnte sie auch nur innerhalb einer Filiation erwähnt sein.

${ }^{32} \ddot{A g}$. Biogr., 494, a.

${ }^{33}$ Kitchen $\left(T I P^{2}, \S \S 178-9\right)$ hatte zunächst einen Sohn 'Hor VIII' als Stifter der Statue angesetzt, aber Bierbrier (Late New Kingdom, 76-8) hat gezeigt, daß es sich höchstwahrscheinlich doch um den Enkel Hor IX handelt. In der zweiten Auflage seines Buches $\left(T I P^{2}, \S 476\right)$ hat sich Kitchen dem angeschlossen. Vgl. auch D. B. Redford, 'New Light on Temple J at Karnak', Or 55 (1986), 7, n. 48.

${ }^{34}$ Oder sogar von Schoschenk 'IV.', vgl. D. Aston, 'Takeloth II-a King of the 'Theban Twenty-Third Dynasty'?', ҰEA 75 (1989), 151-2.

${ }^{35}$ Obwohl eine frühere Aufstellung nicht definitiv ausgeschlossen werden kann (s. Bierbrier, Late New Kingdom, 76), ist sie doch extrem unwahrscheinlich.

${ }^{36} \mathrm{Zur}$ Statue s. meinen Artikel 'Drei Gebete aus der 22. Dynastie' in Form und Mass, Festschrift für G. Fecht, hrsg. J. Osing u. G. Dreyer (ÄUAT 12, Wiesbaden 1987), 238-53; zur Datierung vgl. Bierbrier, Late New Kingdom, 82 (auch nach Astons Chronologie [s.u.] kann die Statue kaum der Zeit Osorkons III. zugewiesen werden) und zur Familie den Stammbaumausschnitt bei Aston, $\mathscr{F E A ~ 7 5 , ~} 145$.
} 
Zudem dient der Titel des Hohenpriesters auf der Statue Kairo CG 42225 als (zusätzlicher) 'Genehmigungsvermerk' (o.ä.), was sonst nahezu ausschließlich Königen vorbehalten ist und bei Harsiese A als König ja auch tatsächlich vorkommt (s.o., Belege G und H). Die einzige Ausnahme findet man auf der Statue Kairo CG 42232. ${ }^{37}$ Dort erscheinen Titel und Namen des Hohenpriesters Harsiese B neben den Kartuschen Schoschenks III.

Dieser Harsiese B ist zuerst im Jahr 6 Schoschenks III. sicher bezeugt, ${ }^{38}$ danach im Jahr 5 Petubastis' I. (= Jahr 12 Schoschenks III.), ${ }^{39}$ in seinem Jahr $8,{ }^{40}$ [18] und $19 .{ }^{41}$ Nach der bis vor kurzem weitestgehend akzeptierten Chronologie der 22./23. Dynastie nach Kitchen könnte der Hohepriester von Kairo CG 42225 kaum Harsiese B sein: Er ist zuletzt im Jahr 19 des Petubastis (entsprechend Jahr 26 Schoschenks III.) belegt. Wenn zwischen Osorkon II. und Schoschenk III. noch Takeloth II. mit etwa 25 Regierungsjahren läge, wäre er (mit Unterbrechungen) über einen Zeitraum von mehr als 50 Jahren Hoherpriester gewesen. Neuerdings hat aber D. Aston völlig überzeugend nachgewiesen, daß Takeloth II. ein König der 'thebanischen 23. Dynastie' war und nicht zur tanitischen Linie der 22. Dynastie gehört. ${ }^{42} \mathrm{Er}$ ist also nicht Nachfolger Osorkons II. und Vorgänger Schoschenks III., sondern diese beiden folgen unmittelbar aufeinander. Takeloth II. gehört zu einer parallelen 'thebanischen' Dynastie und beginnt seine Regierungszeit gegen Ende der Herrschaft Osorkons II., so daß sie weitestgehend der von Schoschenk III. parallel verläuft (Takeloths Jahr 4 entspricht Jahr 1 Schoschenks III.). Das heißt aber, wenn Harsiese B im Jahr 6 Schoschenks III. zuerst belegt ist, wäre es leicht möglich, daß er sein Amt auch schon einige Jahre vorher antrat, am Ende der Regierungszeit Osorkons II. Bei dem Hohenpriester Harsiese von Kairo CG 42225 dürfte es sich also eher um Harsiese B handeln. Mit anderen Worten, Harsiese A ist ausschließlich als König belegt! Natürlich muß das nicht notwendig bedeuten, daß er nicht (vorher) auch Hoherpriester war; das wäre in der Tat gar nicht so unwahrscheinlich, betrachtet man etwa die 'Karrieren' von Osorkon III. und Takeloth III., ist aber bis auf weiteres nicht $\mathrm{zu}$ belegen.

\section{Die thebanischen Hohenpriester unter Osorkon II.}

Wenn aber Harsiese B schon in der Zeit Osorkon II. Hoherpriester war, muß man sich fragen, ob und wie er in dessen Regierungszeit unterzubringen ist. Im Gegensatz zu dem, was Kitchen ${ }^{43}$ und Aston ${ }^{44}$ angenommen haben, besteht in der Zeit Osorkons II. durchaus nicht etwa ein 'Überangebot' an Hohenpriestern des Amun, im Gegenteil, die

${ }^{37}$ Äg. Biogr., 556, c.

${ }^{38}$ Nilstandsmarke Nr. 23, s. J. von Beckerath, 'The Nile Level Records at Karnak and their Importance for the History of the Libyan Period (Dynasties XXII and XXIII)', FARCE 5 (1966), 51.

${ }^{39}$ Nilstandsmarke Nr. 24, ibid., 51.

40 'Priesterannalen', Nr. 2, s. ̈̈g. Biogr., 567.

${ }^{41}$ Nilstandsmarken Nr. 27 [und 28], s. von Beckerath, FARCE 5, 52.

${ }_{42} \mathcal{} E A$ 75, 139-53. Die Einwände von Beckeraths (BiOr 49 (1992), 704, n. 4) halte ich nicht für stichhaltig: Das 'unglaubhafte Nebeneinander' mehrerer Könige ist für die spätere 3. Zwischenzeit in unanfechtbarer Weise bezeugt. Und wenn der Hohepriester Osorkon B nach Schoschenk III. datiert, muß das nichts damit zu tun haben, daß sein Vater zu dessen Linie gehört habe, wie unten gezeigt wird. S. jetzt auch von Beckerath selbst, 'Beiträge zur Geschichte der Libyerzeit', GM 144 (1995), 8.

${ }^{43} T I P^{2}, \S 157 ; 456$.

${ }^{44} \mathfrak{F E A} 75,147$. 


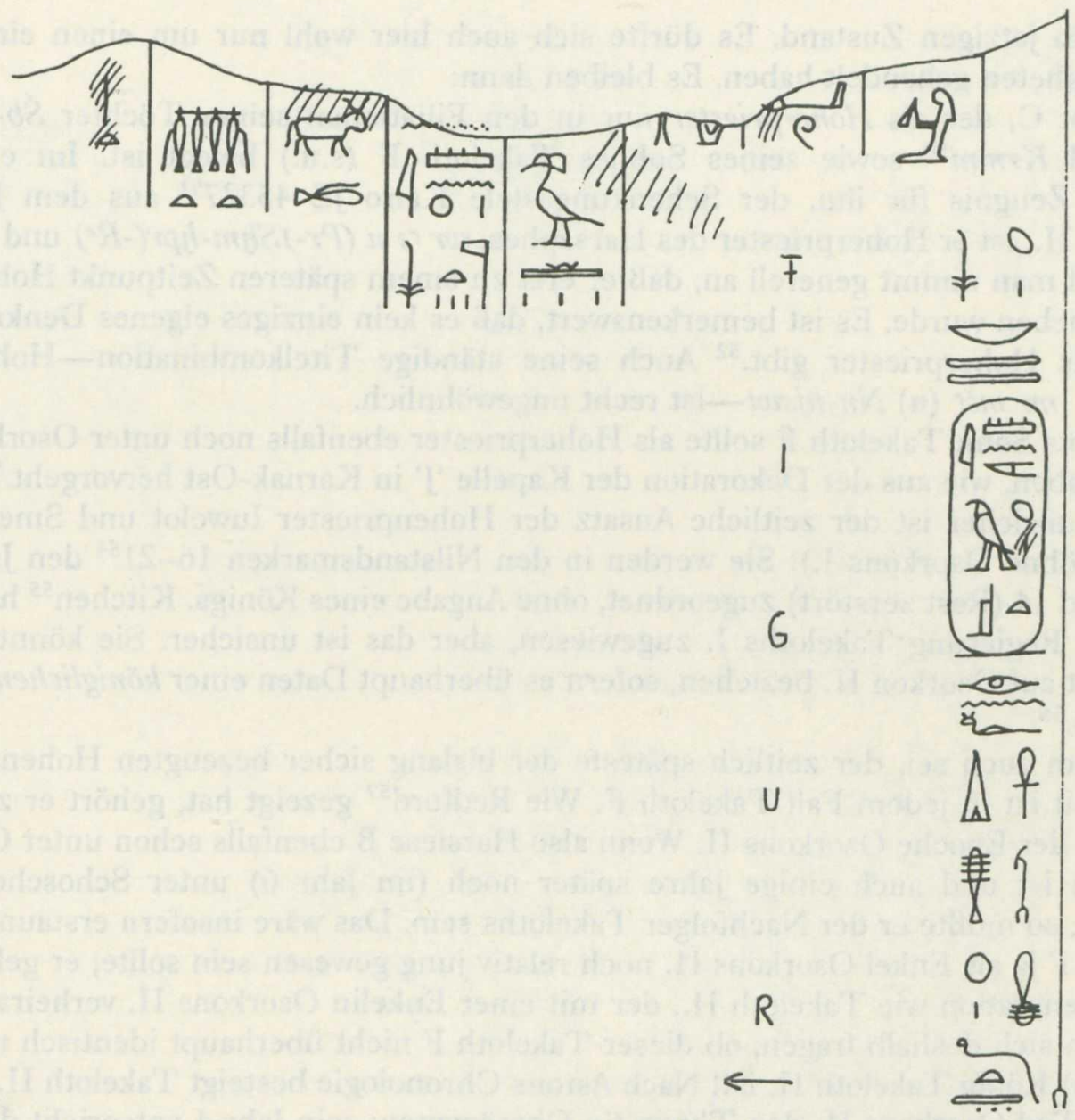

Fig. 4. Kairo JE 37516.

Belege sind eigentlich erstaunlich spärlich: Wie gesehen, entfällt Harsiese A jetzt, zumindest ist er nicht nachweisbar, und den kleinen Hornacht aus Tanis, den Aston mit aufführt, sollte man besser ganz aus dem Spiel lassen.

Ein Sohn des Harsiese A, dessen Namen nicht vollständig erhalten ist (s.o., Beleg A), war Legrain zufolge Hoherpriester des Amun. ${ }^{45}$ Yoyotte spricht dagegen bei demselben Beleg nur von 'un prêtre d'Amonrasonther, fils du roi Harsiésis' ${ }^{46}$ Tatsächlich ist von seinem Titel nur noch [...] $n$ Fmn-Re njswt ntrw erhalten, wie eine Kollation ${ }^{47}$ ergeben hat (vgl. Fig. 4 und Pl. XIII, 1), und eine Ergänzung des Namens ${ }^{48}$ ist nicht möglich, da die Begrenzung der Kolumnen nach oben unklar ist. Natürlich wäre es denkbar, daß der Abbruch über $n$ Fmn-Rr rezent ist und Legrain (und Daressy) noch mehr gesehen hatten, aber das scheint recht unwahrscheinlich: Der Stein ist überall sehr massiv und ohne Sprünge, und die Beschreibung der Stelle durch Daressy entspricht ansonsten

${ }^{45}$ ASAE 6, 124; ebenso Daressy, RecTrav 35, 143.

${ }^{46} \mathrm{EPHE} 86,163$.

${ }^{47}$ Heutiger Standort im Museum Kairo: R 49 centre N.

${ }^{48} \mathrm{Zu}$ Ergänzungsvorschlägen s. Daressy, RecTrav 35, 143; von Beckerath, Handbuch, 104, n. 11; TIP ${ }^{2}$, $\S 157,6$. 
genau dem jetzigen Zustand. Es dürfte sich auch hier wohl nur um einen einfachen Amunpropheten gehandelt haben. Es bleiben dann:

(a) Nimlot C, der als Hoherpriester nur in den Filiationen seiner Töchter $\breve{S} b-n$-Spdt $(A)^{49}$ und $\mathrm{Krmm}^{50}$ sowie seines Sohnes Takeloth F (s.u.) belegt ist. Im einzigen datierten Zeugnis für ihn, der Schenkungsstele Kairo JE $45327^{51}$ aus dem Jahr 16

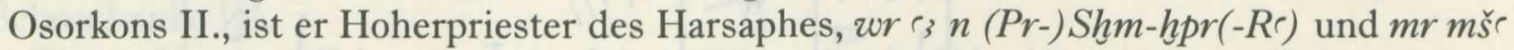
$h_{3} w t j$, und man nimmt generell an, daß er erst zu einem späteren Zeitpunkt Hoherpriester in Theben wurde. Es ist bemerkenswert, daß es kein einziges eigenes Denkmal des Nimlot als Hoherpriester gibt. ${ }^{52}$ Auch seine ständige Titelkombination-Hoherpriestertitel $+m r m s ̌ r(n) N n$-njswt-ist recht ungewöhnlich.

(b) Nimlots Sohn Takeloth F sollte als Hoherpriester ebenfalls noch unter Osorkons II. amtiert haben, wie aus der Dekoration der Kapelle 'J' in Karnak-Ost hervorgeht. ${ }^{53}$

(c) Sehr unsicher ist der zeitliche Ansatz der Hohenpriester Iuwelot und Smendes C (beides Söhne Osorkons I.): Sie werden in den Nilstandsmarken $16-21^{54}$ den Jahren 5 bzw. 8 und 14 (Rest zerstört) zugeordnet, ohne Angabe eines Königs. Kitchen ${ }^{55}$ hat diese Jahre der Regierung Takeloths I. zugewiesen, aber das ist unsicher. Sie könnten sich ebensogut auf Osorkon II. beziehen, sofern es überhaupt Daten einer königlichen Regierung sind. ${ }^{56}$

Wie dem auch sei, der zeitlich späteste der bislang sicher bezeugten Hohenpriester dieser Zeit ist in jedem Fall Takeloth $\mathrm{F}$. Wie Redford ${ }^{57}$ gezeigt hat, gehört er ziemlich ans Ende der Epoche Osorkons II. Wenn also Harsiese B ebenfalls schon unter Osorkon II. belegt ist und auch einige Jahre später noch (im Jahr 6) unter Schoschenk III. erscheint, so müßte er der Nachfolger Takeloths sein. Das wäre insofern erstaunlich, als Takeloth F ja als Enkel Osorkons II. noch relativ jung gewesen sein sollte; er gehört zur selben Generation wie Takeloth II., der mit einer Enkelin Osorkons II. verheiratet war. Man kann sich deshalb fragen, ob dieser Takeloth $\mathrm{F}$ nicht überhaupt identisch mit dem (späteren) König Takeloth II. ist! Nach Astons Chronologie besteigt Takeloth II. 3 Jahre vor dem Tod Osorkons II. den Thron (in Oberägypten; sein Jahr 4 entspricht dem Jahr 1 Schoschenks III.). Wäre er identisch mit Takeloth $\mathrm{F}$, würde das die Tatsache erklären, daß dieser-obgleich noch nicht alt-nur einmal belegt ist und dann spurlos verschwindet. Ferner ist Takeloth II. nach Aston ein thebanischer (oberägyptischer) König, und von daher wäre es gar nicht unwahrscheinlich, daß er vorher Hoherpriester war, wie das ja zumindest für Osorkon III. ${ }^{58}$ und Takeloth III. gesichert ist.

\footnotetext{
${ }^{49}$ Kairo CG 42228, f; g; m; n, s. Äg. Biogr., 520; 521; 524; Kairo CG 42229, d, s. ibid., 552; Kairo CG 18496, s. Quibell und Spiegelberg, Ramesseum, pl. xxvii.

${ }^{50}$ Oriental Institute of Chicago, The Epigraphic Survey, Reliefs and Inscriptions at Karnak, III. The Bubastite Portal (Chicago 1954), pl. 17.

${ }^{51}$ E. Iversen, Two Inscriptions Concerning Private Donations to Temples (Kopenhagen 1941), $1 \mathrm{ff}$.

${ }^{52}$ Und auf der Pasenhorstele (Z. 8) wird er nur als $\langle z 3\rangle$ njswt mj $n n$ bezeichnet.

${ }^{53} \mathrm{Kees}$, Hohenpriester, 113; Redford, Or 55, 14.

${ }^{54}$ Von Beckerath, $7 A R C E$ 5, 50-1.

${ }^{55} T I P^{2}, \S \S 96 ; 157$.

${ }^{56}$ Es wäre m.E. grundsätzlich keineswegs ausgeschlossen, daß es sich um Regierungsjahre dieser Hohenpriester selbst handelte: Das ist bei unvoreingenommener Betrachtung der Texte sogar die naheliegendste Lösung, wenn es auch dafür in der 22. Dynastie offenbar keine Parallele gibt.

${ }^{57}$ Or $55,14$.

${ }^{58} \mathrm{~S}$. The Paleological Association of Japan, Preliminary Report. Second Season of the Excavations at the Site of Akoris, Egypt 1982 (Kyoto 1983), 13-14 pl. 11. Darüber hinaus dürfte Osorkon III. ohne jeden Zweifel mit dem vormaligen Hohenpriester Osorkon B identisch sein, s. Aston, $\mathscr{F E A ~ 7 5 , 1 5 0 .}$
} 
Das einzige Problem bei dieser Lösung bestünde darin, daß er dann mit seiner Schwester (oder Halbschwester) Karomama (einer Tochter von Nimlot C) verheiratet gewesen sein müßte, aber das ist kein unüberwindliches Hindernis: Zum einen sind Geschwisterehen in Königsfamilien ohnehin nichts Besonderes; für die 21. Dynastie wird dasselbe z.B. im Fall Psusennes I./Mutnedjemet angenommen..$^{59}$ Und zudem sind gerade aus dieser Zeit, unter den libyschen Magnaten, auch Fälle außerhalb der engeren Königsfamilie bekannt: Ein Hoherpriester von Memphis und Urenkel Osorkons II. ist mit seiner Schwester (oder Halbschwester) verheiratet, ${ }^{60}$ und auch zwei Kinder eines Wezirs $n h$-Wsrkn (ein Enkel Osorkons III.) heiraten einander. ${ }^{61}$

Wenn Takeloth F also wenige Jahr vor dem Tod Osorkons II. selbst König wurde, ist der Platz frei für Harsiese B: Er dürfte in den letzten 3 Jahren Osorkons II. in der Nachfolge von Takeloth $\mathrm{F}$ als Hoherpriester amtiert haben, und die Statue Kairo CG 42225 wird aus eben dieser Zeit stammen. ${ }^{62}$

\section{Die Anfänge der 'thebanischen' 23. Dynastie}

Es stellt sich nun die Frage, ob und wie diese Vorschläge in das Gesamtbild der thebanischen 23. Dynastie einzupassen sind, aufbauend auf der von Aston ${ }^{63}$ dargelegten Chronologie: Schoschenk III. folgt (in Tanis) unmittelbar auf Osorkon II.; sein Jahr 1 entspricht dem Jahr 4 Takeloths II. (in Theben), und sein Jahr 8 ist das Jahr 1 Petubastis' I. und das Jahr 11 Takeloths II. Da in diesem letzteren Jahr eine Rebellion in Theben losbricht, ist es naheliegend, diese (mit Aston) mit der Thronbesteigung des Petubastis zu verbinden. Es gibt also offenbar in Oberägypten zwei rivalisierende Königshäuser, in dieser Zeit durch Takeloth II. und Petubastis vertreten. Beide Häuser stellen offenbar nicht nur je einen König, sondern dazu auch noch einen Hohenpriester: Die Zuordnung von Osorkon B zu Takeloth II. ergibt sich aus den 'Osorkonannalen', ist aber ohnehin klar, da es sich um seinen ältesten Sohn handelt. Dagegen datiert der Hohepriester Harsiese B nach dem Regierungsantritt des Petubastis immer nach diesem; er wird also dessen 'Fraktion' zugehören. Der Nachfolger in dieser Funktion ist offenbar Takeloth $\mathrm{E}:{ }^{64} \mathrm{Er}$ schließt einerseits chronologisch direkt an Harsiese $\mathrm{B}$ an, ${ }^{65}$ während andrerseits Osorkon B noch im Jahr 39 Schoschenks III. gleichfalls als Hoherpriester bezeugt ist; also kann Takeloth E nicht zu dieser Partei gehört haben.

Wie Aston ${ }^{66}$ zuletzt noch einmal deutlich gemacht hat, dürfte es doch einen Scho-

${ }^{59} \mathrm{Vgl} . T I P^{2}, \S 40$.

${ }^{60}$ S. J. Černý, 'Consanguineous Marriages in Pharaonic Egypt', $\mathscr{} E A 40$ (1954), 23; TIP ${ }^{2}, \S 155$ n. 23.

${ }^{61}$ Vgl. M. L. Bierbrier, 'Two Confusing Coffins', $\mathscr{F E A} 70$ (1984), 84 (Hinweis eines Referee des $\mathcal{Y E A}$ ).

${ }^{62}$ Nach Einreichen des Manuskripts dieses Aufsatzes hat N. Dautzenberg, 'Bemerkungen zu Schoschenq II., Takeloth II. und Petubastis II.', GM 144 (1995), 24-6, gleichfalls vorgeschlagen, Takeloth II. mit dem Hohenpriester Takeloth $\mathrm{F}$ gleichzusetzen. Daraus ergibt sich auch für ihn, daß der Amtsantritt von Harsiese B vor dem von Osorkon B liegen muß.

${ }^{63}$ FEA 75, 148-9. Die Verschiebung um ein Jahr (Jahr 1 Schoschenks III. = Jahr 5 Takeloths II.), die J. von Beckerath neuerdings vorgeschlagen hat (GM 144, 9-11), überzeugt nicht. Sie basiert wohl auch nur auf dem angeblichen, aber ganz unwahrscheinlichen Jahr 26. Takeloths II. (s.u., n. 72).

${ }^{64}$ Von Kees (Hohenpriester, 138-40) und Kitchen $\left(T I P^{2}, \S 163\right)$ zu Unrecht mit Takeloth F identifiziert, vgl. Redford, Or 55, 14 n. 89, und Aston, FEA 75, 147.

${ }^{65}$ Letztes Datum für Harsiese B: Jahr 19 Petubastis' I., erstes für Takeloth E: Jahr 23 derselben Regierung.

6 YEA 75, 151-2. 
schenk IV. gegeben haben ${ }^{67}$ und da Takeloth $\mathrm{E}$ nach diesem Schoschenk datiert, dürfte es sich um den Nachfolger des Petubastis handeln. Es bleibt dann noch der König Iuput einzuordnen, dessen Jahr 2 in der Nilstandsmarke Nr. $26^{68}$ dem Jahr 16 Petubastis' I. gleichgesetzt wird. Aufgrund dieser Gleichsetzung wird er meist als Koregent des Petubastis eingestuft, bei Kitchen zunächst noch mit einer eigenen 10jährigen Regierungszeit, ${ }^{69}$ in der 2 . Auflage dann ${ }^{70}$ nur noch als kurzfristiger Mitherrscher ohne eigene Jahre. A. Leahy ${ }^{71}$ und D. B. Redford ${ }^{72}$ haben aber zurecht betont, daß die chronologische Gleichsetzung keineswegs bedeuten muß, Iuput sei Koregent des Petubastis gewesen. Die verfügbaren Daten suggerieren vielmehr eine andere Lösung: Wenn Iuputs Jahr 2 dem Jahr 16 des Petubastis entspricht, fällt sein 1. ins 25. Jahr Takeloths II. und ins 22. Jahr Schoschenks III. (nach Astons Chronologie). Nun ist Jahr 25 das letzte für Takeloth II. bezeugte ${ }^{73}$ und es muß zugleich sein Todesjahr sein, da in den 'Osorkonannalen' unmittelbar an das Jahr 24 Takeloths II. das Jahr 22 Schoschenks III. schließt. ${ }^{74}$ Es liegt also nichts näher, als daß Iuput tatsächlich der Nachfolger Takeloths II. war und kein Koregent des Petubastis. Dafür spricht noch etwas anderes: Nach der früher akzeptierten Chronologie folgte Schoschenk III. mit einer langen Regierungszeit auf Takeloth II., und deshalb war es klar, wieso der Hohepriester Osorkon B trotz seiner Ansprüche als Kronprinz nicht seinem Vater auf den Thron folgen konnte, sondern Hoherpriester blieb. ${ }^{75}$ Nach Astons Chronologie entfällt diese Annahme, und man fragt sich, wieso Osorkon B nun nicht Nachfolger wurde, denn kurz vor dem Tod seines Vaters ist er (im Jahr 24) nach fast 10jährigem 'Exil' ja wieder in Theben. Diese Anwesenheit geht, wie seinem eigenen Bericht $\mathrm{zu}$ entnehmen ist, ${ }^{76}$ auf eine gütliche Einigung der Parteien zurück. ${ }^{77}$ Bei dieser Lage der Dinge bietet sich folgendes Szenario von selbst an: Die gütliche Einigung bestand eben darin, daß Osorkon B (vorläufig zumindest) auf den Thron verzichtete, den ein offenbar akzeptablerer Kandidat, eben Iuput I., bekam, wofür aber wiederum Osorkon B in Theben als Hoherpriester akzeptiert wurde.

Die Einigung muß einige Zeit vor dem Tod Takeloths II. erzielt worden sein, denn, wie gesagt, Osorkon ist schon in dessen vorletztem Jahr (24) wieder in Theben mit Stiftungen belegt. Grund für den Kompromiß dürfte also der absehbare Tod des Takeloth gewesen sein, und dies läßt sich tatsächlich expressis verbis der 'Osorkonchronik' entnehmen: In B, Z.8 sagt Osorkon zu seinen Gefolgsleuten: $n$ r his.th $h$ hr mnj.f $p w$. Caminos $^{78}$ übersetzt 'You shall not fight, because (your fighting) would be the death of him' und sieht darin eine metaphorische Ausdrucksweise. Wörtlich heißt es aber eigentlich 'ihr sollt nicht kämpfen, denn es ist so $(p w)$, daß er stirbt', und das muß keine

\footnotetext{
${ }^{67}$ So auch TIP 2 , 68 und Bonhême, Noms royaux, 124-8; von Leahy ('Abydos in the Libyan Period', Libya and Egypt, c1300-750 BC, hrsg. Leahy (London 1990), 183) und mir selbst (BiOr 47 (1990), 87) wohl zu Unrecht bestritten. Vgl. auch von Beckerath, BiOr 49, 704.

${ }^{68}$ Von Beckerath, FARCE 5, 52.

${ }^{69}$ TIP ${ }^{2}$, $302 ;$ p. 467.

${ }^{70}$ TIP ${ }^{2}, \S 448 ;$ p. 588.

${ }^{71}$ Libya and Egypt, 183.

${ }^{72}$ Pharaonic King-Lists, Annals and Day-Books (Mississauga 1986), 316 n. 125.

${ }^{73}$ Das Jahr 26, das ihm Kitchen $\left(T I P^{2}, \S 294\right)$ zuweisen möchte, wird zu Schoschenk III. gehören.

${ }^{74}$ The Epigraphic Survey, Reliefs III, pl. 22, Z.7-12.

${ }^{75}$ Vgl. etwa $T I P^{2}$, § 294, II. Nach Einreichen des Manuskripts dieses Aufsatzes hat sich auch J. von Beckerath (GM 144, 10-11) dafür ausgesprochen, daß Iuput I. der Nachfolger Takeloths II. ist.

${ }^{76}$ Osorkonchronik (s.u.), B, Z. 7 ff.

${ }^{77} \mathrm{Im}$ Text als durch göttliche Intervention ermöglicht dargestellt.

${ }^{78}$ R. A. Caminos, The Chronicle of Prince Osorkon (AnOr 37, Rom 1958) (im folgenden CPO), § 136.
} 
Metapher sein. Wie dem auch sei, auf jeden Fall ist es nicht überraschend, daß Osorkon in seinem Bericht in der Folgezeit nur nach Schoschenk III. datiert, ${ }^{79}$ denn man wird ja davon ausgehen können, daß er sich selbst weiterhin als den legitimen Erben ansah.

\section{Die Osorkonchronik}

Dieses Verständnis der Dinge hat den Vorzug, daß auf diese Weise sich die erhaltenen Daten zu einem sinnvollen Bild zusammenfügen, daß sie sich gegenseitig stützen und bestätigen und daß vor allen Dingen eine fast perfekte Übereinstimmung mit den in der 'Osorkonchronik' berichteten oder angedeuteten Geschehnissen erreicht wird (vgl. zum Folgenden Übersicht und Belegstellen in Tab. 1, p. 149). Dieser Text beginnt im Jahr 11 Takeloths II. (= Jahr 8 Schoschenks III.), und zu Beginn ist Osorkon B offenbar noch nicht Hoherpriester, wie Kees sehr richtig gegen Caminos betont hat: ${ }^{80}$ Die in A, 25-6 geschilderten Riten gehören ganz offensichtlich zur Amtseinführung Osorkons, ${ }^{81}$ die dann durch ein Orakel des Amun sanktioniert wird.

Bei der Schilderung der Ausgangslage (A, 19-22) wird gesagt, daß Osorkon mit dem Stand der Dinge unzufrieden ist, wenn auch nicht aus unbefriedigtem Ehrgeiz ( $n \underline{d} d$.f ' $3 . j$ jm' 'er sagte nicht "ich möchte dadurch bedeutend werden"'), und daß er Amun seit jeher sehr ergeben war. Das kann in dieser Kombination nur bedeuten, daß er unzufrieden damit ist, nicht Hoherpriester zu sein. Danach, als eine thebanische 'Rebellion' ausbricht, zweifellos eine Umschreibung für die Thronbesteigung des Petubastis, ${ }^{82}$ greift Osorkon auf Wunsch des Harsaphes ein, das wird wohl heißen, nachdem er sich dessen Einverständnis durch ein Orakel versichert hat. ${ }^{83}$ Dann wird kurz von Unterdrückung von Feinden sowie von Baumaßnahmen in Hermopolis (! s.u.) berichtet, bevor Osorkon nach Theben fährt, sich dort als Hoherpriester etabliert, die Rebellen bestraft und verschiedene Dekrete erläßt.

Im Jahr 12 (B,1) ist Osorkon offenbar unangefochten, im Jahr 15 aber bricht eine Rebellion aus, gefolgt von einem jahrelangen Bürgerkrieg $(B, 7)$. Diese Rebellion muß der Rückkehr des Harsiese B zusammenhängen, der in diesem Jahr und einigen folgenden wieder in Theben belegt ist. Bedeutend später, wohl im 24. Jahr Takeloths II. (s.o.) kommt es zu der geschilderten gütlichen Einigung (und kurz darauf zur Thronbesteigung Iuputs I.). Wie lange Iuput amtiert hat, ist völlig ungewiß. Auf jeden Fall hat die Einigung in Theben nicht lange Bestand gehabt, denn zwischen den Jahren 25 und 28 Schoschenks III. klafft in den Stiftungsaufzeichnungen der Osorkonchronik eine Lücke, und zugleich ist Harsiese B in den Jahren 18 und 19 des Petubastis (=25/26 Schoschenks III.) wieder als Hoherpriester in Theben belegt. Also hat er im Verlauf des Jahres 25 Schoschenks III. Osorkon erneut verdrängt, und dieser Vorgang dürfte der zweiten Revolte in der Osorkonchronik entsprechen. ${ }^{84}$ Nach nicht allzulanger Zeit kann aber Osorkon sein Amt wieder in Theben ausüben. Obwohl der entsprechende Abschnitt der Osorkonchronik (C, Z.2ff.) lückenhaft ist, läßt sich ihm entnehmen, daß auch

\footnotetext{
${ }^{79}$ Während zur gleichen Zeit (im Jahr 23 Shoschenks III.) der Nilstand (Inschrift Nr. 26) nach Iuput und Petubastis markiert wird, sicher eben als Folge des Kompromisses.

${ }^{80}$ Hohenpriester, $117-18$.

${ }^{81}$ Vgl. dazu auch J. Leclant, 'La Chronique du Prince Osorkon', Or 30 (1961), 409-10, und M. Römer, Gottes- und Priesterherrschaft in Ägypten am Ende des Neuen Reiches (ÄUAT 21, Wiesbaden 1994), 484.

${ }^{82} \mathrm{~S}$. Aston, $\mathscr{F} E A 75,148-9$.

${ }^{83}$ Von Caminos $(C P O, \S 41 \mathrm{f})$ nicht als Orakel, sondern als Traumoffenbarung gedeutet.

${ }^{84}$ The Epigraphic Survey, Reliefs III, pl. 22, Z. 1-2; CPO, § 172. Man beachte, daß in Herakleopolis, dem
} 
diesmal die Einigung friedlich war, und desgleichen war angeblich wieder göttliche Intervention im Spiel. Auf jeden Fall ist die gute Nachricht, die aus Theben kommt ('be happy, you have no enemies') unschwer zu erraten: Es kann sich nur um den Tod des Harsiese handeln, der danach nicht mehr in den Quellen erscheint.

Im Jahr 28 und 29 ist dann allein Osorkon in Theben belegt (und die Osorkonchronik endet hier, Jahr 29 ist nur noch als Nachtrag verzeichnet), aber im Jahr 23 des Petubastis (= Jahr 30 Schoschenks III.) erscheint ein Hoherpriester Takeloth, der offenbar den Kurs des Harsiese fortsetzt (sein Sohn?). Dieser Takeloth ist dann auch noch unter Schoschenk IV., dem Nachfolger des Petubastis, belegt, und zwar im Jahr 6 (das ins Jahr 35-39 Schoschenks III. fallen muß), während Osorkon erst im 39. Jahr Schoschenks III. wieder auf der Bühne erscheint, zusammen mit seinem Bruder, dem General Bakenptah, nachdem sie 'alle, die gegen sie kämpften, niedergeworfen hatten'. In diesem Jahr oder kurz danach dürfte der Hohepriester Osorkon B dann als Osorkon III. selbst den Thron bestiegen haben.

Es ist übrigens bemerkenswert, daß es trotz allem Hin und Her und aller Kämpfe (die ja bei jedweder Interpretation unzweifelhaft sind) offenbar keinerlei damnatio memoriae gegeben hat. Soweit sich das beurteilen läßt, ist kein einziger Name der rivalisierenden Parteien entfernt oder bewußt beschädigt worden. Das dürfte dafür sprechen, daß alle Seiten diesen Streit als etwas vielleicht zeitweise Unangenehmes ansahen, aber letztlich doch als verständlichen und legitimen Wettstreit innerhalb der Fürstenfamilien, also ganz im feudalen libyschen Verständnis, und nicht in ägyptischer Tradition als Kampf zwischen Ordnung und Chaos.

\section{Petubastis I.}

Bei all diesen Ereignissen spielt der König Petubastis eine wichtige Rolle. Als Osorkon B im Jahr 11 seines Vaters die erste thebanische 'Rebellion' niederwirft, bei der es sich eben um die Thronbesteigung dieses Petubastis handeln wird, ist folgendes überraschend: In seiner Schilderung der Ereignisse ist die Fahrt nach Theben ganz friedlich (A, Z.24-5), die gewaltsamen Ereignissen haben sich offenbar weiter nördlich in oder bei Hermopolis abgespielt. Natürlich wäre es möglich, daß dort (mehr oder weniger zufällig) die Entscheidungsschlacht stattfand, wie Caminos angenommen hat. ${ }^{85}$ Aber auch eine andere Lösung wäre denkbar: Gegen Ende der 3. Zwischenzeit sind auch Könige von Hermopolis bekannt: einmal Thotemhab, ${ }^{86}$ v.a. aber Nimlot D, der als Verbündeter des Tefnacht und Gegner des Pianchi in der Pianchistele eine prominente Rolle hat. Es wäre nicht ausgeschlossen, daß eben diese Königslinie schon auf Petubastis zurückgeht und daß die thebanische Sezession darin bestand, einen in Hermopolis regierenden König anzuerkennen.

Wie Leahy, ${ }^{87}$ gefolgt von Aston ${ }^{88}$ festgestellt hat, ist Petubastis I. ein 'thebanischer'

militärischen Schwerpunkt von Osorkon B, weiter nach Schoschenk III. datiert wird: Auf einer Schenkungsstele der Sammlung Michailides aus Herakleopolis ist das Jahr 26 (entsprechend dem Jahr 19 Petubastis' I.) belegt, s. D. Meeks in State and Temple Economy in the Ancient Near East, hrsg. E. Lipiński, II (OLA 6, Löwen 1979), 669 [22.8.26].

${ }^{85} \mathrm{CPO}, \S 284$.

${ }^{86}$ Vgl. TIP 2 , 3331 ; P. A. Spencer und A. J. Spencer, 'Notes on Late Libyan Egypt', $\mathscr{F E A} 72$ (1986), 198-9; H. Wild, 'Une statue de la XII ${ }^{\mathrm{e}}$ dynastie utilisée par le roi Hermopolitain Thot-em-hat de la XXIII' ${ }^{\mathrm{e}}, R d E$ 24 (1972), 209-15.

${ }^{87}$ Libya and Egypt, 182-3.

${ }^{88} \mathrm{FEA} \mathrm{75,} 149$. 
König. ${ }^{89}$ Dennoch bleiben bei Petubastis (anders als etwa bei Takeloth II.) einige Belege aus Unterägypten übrig, die nicht so einfach wegzudiskutieren sind..$^{90}$ V.a. aus einigen Schenkungsstelen muß man schließen, daß in Unterägypten zumindest nach Petubastis datiert werden konnte.

Zudem können auch die Königsnamen des Petubastis eine typische 'unterägyptische' Form zeigen: In der 3. Zwischenzeit führen viele Könige ein Beiwort z3 Bsstt oder z 3 sst (z.T. $+n \underline{t} r \underline{h q_{3}} W_{3 s t}$ ) in ihren Kartuschennamen. ${ }^{91}$ Bei Schoschenk I. schienen bislang beide Varianten sicher bezeugt, ${ }^{92}$ aber später haben fast alle Könige (wenn überhaupt) nur eines von beiden, so daß man z.B. Osorkon II. und III. an diesen Beiworten sicher unterscheiden kann. ${ }^{93}$ Wie zuletzt Yoyotte gezeigt hat, ${ }^{94}$ kommt das Beiwort $z 3$ ist v.a. oberägyptischen Königen zu, nämlich Takeloth II., Osorkon III. Takeloth III. und Ұny, während $z_{3}$ B 3 stt v.a. bei unterägyptischen belegt ist: Osorkon II., Schoschenk III., Pami, Schoschenk V. und Iuput II. Auch bei Schoschenk I. fände man z 3 st bei einem oberägyptischen, $z_{3}$ Bistt bei einem unterägyptischen Beleg. Bei Petubastis I. trifft man nun auffälligerweise beide Beiworte. ${ }^{95}$ Man könnte versucht sein, die Belege auf zwei Könige zu verteilen: ${ }^{96}$ Petubastis Stp. $n$ - $7 m n$ z 3 sst in Oberägypten und Petubastis Stp.n$F_{m n} z_{3} B_{3}$ stt in Unterägypten, aber dafür gibt es keinerlei seriöse Anhaltspunkte. Es dürfte nur einen Petubastis Stp. $n$ - $¥ m n$ geben, der sich in Unterägypten $z ; B$ Bstt und in Oberägypten $z_{3}$ 3st nannte. Das wird sicher nicht bedeuten, daß Petubastis ein in Unterägypten herrschender König war (das hätte das Haus Takeloths II. wohl wenig gestört), aber man sollte daraus doch schließen, daß er dort einige Unterstützung genoß.

Darauf deutet auch eine Bauinschrift eines Sohnes Schoschenks III., $P_{3}-\check{s} d j-B_{3} s t t$, am 10. Pylon in Karnak hin, ${ }^{97}$ die nach Petubastis I. datiert ist. Und noch etwas ist für diese Frage von Bedeutung: Auf seiner Statue Kairo CG $42226^{98}$ spricht der bekannte königliche Sekretär Hor IX von seinen Tätigkeiten für den König, und er geriert sich dabei wie dessen spiritus rector, ${ }^{99}$ ja sein Ton hat geradezu etwas Herablassendes. ${ }^{100}$ Da die Statue die Kartuschen des Petubastis trägt, liegt es nahe, diese Sätze auch auf Petubastis zu beziehen, zu dessen 'Partei' Hor IX deshalb gehört haben sollte. ${ }^{101}$

\footnotetext{
${ }^{89}$ Man sollte vielleicht besser - auch ungeachtet der obigen Ausführungen-in diesen Fällen generell von 'oberägyptischen' Königen sprechen, denn es ist ja auch keineswegs sicher, wo Takeloth II. und später Osorkon III. und Takeloth III. ihre Residenz (und ihren Begräbnisplatz!) hatten.

${ }^{90}$ S. Leahy, Libya and Egypt, 182-3.

${ }^{91} \mathrm{Vgl}$. Bonhême, Noms royaux, 268-71.

${ }^{92}$ Ibid., 105-6. Vgl. jetzt aber A. Dodson, 'A New King Shoshenq Confirmed', GM 137 (1993), 53-8, bes. 55-6.

${ }^{93}$ TIP 2 , $\S 70-3$.

${ }^{94}$ 'Pharaon Jny, un roi mystérieux du VIII ${ }^{\mathrm{e}}$ siècle avant J.-C.', CRIPEL 11 (1989), 123-5.

${ }^{95} \mathrm{~S}$. Bonhême, Noms royaux, 207-8.

${ }^{96}$ Wie es A. Schulman, 'A Problem of Pedubasts', $7 A R C E 5$ (1966), 37-8 getan hat, ebenso neuerdings J. von Beckerath, 'Beiträge zur Geschichte der Libyerzeit', GM 147 (1995), 9-13.

${ }^{97}$ Legrain, 'Au pylône d'Harmhabi à Karnak (X $\mathrm{X}^{\mathrm{e}}$ pylône)', ASAE 14 (1914), 14; 39; vgl. TIP2 § 299.

${ }^{98} \ddot{A g}$. Biogr., 506, c; 510, h.

${ }^{99}$ Vgl. auch Redford, Or 55, 8-9.

${ }^{100}$ Vgl. Äg. Biogr., 138; 506: 'Daß ich Oberhaupt im Palast war, ein Führer der Untertanen, Myrrhe war es für das Herz meines Herrn. Wenn er meine Zunge hörte, lebte er. Ich leitete den König zum Wohl der Beiden Ufer, ich fuhr auf dem Wasser des Gottes. Entsprechend meinen Vorstellungen brachte er die Beiden Länder in Ordnung.'

${ }^{101}$ Vgl. auch Aston, $\mathscr{Y E A} 75,149$. Auch die Herkunft dieses Ratgebers spricht natürlich dafür, daß Petubastis grundsätzlich ein oberägyptischer König war.
} 
Für diesen Hor IX gibt es nun außer den bisher bekannten Belegen ${ }^{102}$ noch ein weiteres Zeugnis, das - jedenfalls als historisches Dokument-noch nicht beachtet worden ist. ${ }^{103}$ Es handelt sich um die Basis einer Statue, die in Mitrahina gefunden worden und von $\mathrm{H}$. Brugsch in seinem Thesaurus (p. 1065) veröffentlicht worden ist. ${ }^{104}$ Brugsch hat den Text aber gründlich mißverstanden, wie aus seiner Überschrift ('Fussgestell einer zertrümmerten Königs-Statue') und seiner falschen Zeilennumerierung hervorgeht. Tatsächlich sind die senkrechten Kolumnen natürlich rückläufig zu lesen, und aus ihnen geht hervor, daß es sich um eine Statue des Ptah, nicht des Königs, handeln muß (Z.3 ['13']: nbj.j tjt $n t$ nfr-hr ). Es ist deshalb zu befürchten, daß Brugschs Abschrift nicht sehr zuverlässig ist, und der jetzige Aufbewahrungsort des Torsos ist leider unbekannt. ${ }^{105}$

Immerhin ist der Stifter der Statue eindeutig Thebaner (Z.1: hm-nț $n$ Fmn $m$ Fpt-swt; Z.8-9 ['8-7']: hwoj.k mr.k $m$ rmțtw hmm-ntr zs wrbw c3w $m$ ch. $k n$ Fpt-swt 'mögest du deinen Freund unter den Menschen schützen, den Propheten, den Sohn großer WebPriester in deinem Palast von Karnak'; vgl. auch Z. 11 ['5']), und den Titeln zufolge (der letzte vor dem Namen ist natürlich žs šct $n$ pr-rз zu lesen) dürfte es sich um Hor IX handeln. Auch die Phraseologie der Inschrift spricht (soweit erkennbar) für eine Datierung in die 22.-23. Dynastie und sogar für Hor IX als Autor: Die Ausdrücke $m r$ 'Freund' ${ }^{106}$ und $h$ st $_{\text {-jb }}$ 'Kummer'107 sind gerade in dieser Zeit sehr gängig, ${ }^{108}$ aber vor allem das Wort $\overline{\overline{\hat{0 ิ O}}}$ 'Nachkommen' in Z. 13 ['3'] ist typisch für diese Epoche. ${ }^{109}$ SchlieBlich kommt in Z. 14 ['2'] ein Verb 品 $s(3) p$ 'bauen, anlegen' vor, das in älterer Zeit nur einmal (in Beni Hassan $=U r k$. VIII, 35, 2) belegt ist und in spätester Zeit dann wieder als sjpj ( $\mathrm{Wb}$ IV, 36-7) auftaucht. Dazwischen kommt es-abgesehen von dem hier besprochenen Beleg-nur ein einziges Mal vor, und zwar ausgerechnet auf der Statue der Frau des Hor IX, in einer Inschrift, die zweifellos von ihrem Mann verfaßt worden sein dürfte. ${ }^{110}$

Alles in allem kann es daher kaum zweifelhaft sein, daß auch diese Inschrift von Hor IX stammt, und damit wäre auch ein wichtiger Helfer des Petubastis in Memphis bezeugt. Die naheliegendste Erklärung all dessen ist wohl, daß sich Petubastis (und Hor IX) in Unterägypten Verbündete gesucht haben, daß sich also eine Koalition gegen

${ }^{102}$ Aufgezählt von Redford, or 55, 6-7.

${ }^{103}$ Es wird aber von I. Pomorska, Les flabellifères à la droite du roi en Egypte ancienne (Warschau 1987), 222, dem Hor IX zugeordnet. Ein weiteres(?) bisher unbekanntes Denkmal des Hor IX ist der Torso A.C. 850 im Musée Vivant Denon, Chalon-sur-Saône, publiziert im Katalog Les collections égyptiennes dans les Musées de Saône-et-Loire (Autun 1988), 78-9.

${ }^{104}$ Vorher schon von A. Mariette, Monuments divers (Paris 1872) pl. 28a (und p. 7) in offenbar etwas schlechterer Form. Die beiden Abschriften sind in PM als Texte verschiedener Denkmäler registriert, vgl. PM III ${ }^{1}, 222$ (Mariette, Mon. div., pl. 28a) = 227 (Thes., 1065); ebenso PM III ${ }^{2}, 848=866$.

${ }^{105}$ Nach freundlicher Auskunft von Herrn Dr J. Malek, Oxford (Brief vom 4. 6. 1993). Übrigens ist auch auf dem in n. 103 erwähnten Fragment im Musée V. Denon vom Errichten von Statuen die Rede, und im gleichen Zusammenhang (Z. 1) wird der Begriff $n f r$ - hr (Kontext zerstört) erwähnt. Es wäre nicht ausgeschlossen, daß beide Bruchstücke tatsächlich zu einer einzigen Statue gehörten. Auf beide Texte werde ich in einem in $S A K 22$ erscheinenden Aufsatz näher eingehen.

${ }^{106}$ Waagerechte Zeile und Z. 7. ['9'].

${ }^{107}$ Ibid.

${ }^{108}$ hst-jb z.B. in Äg. Biogr., 444, Z. 7; 495, Z. 7; Jansen-Winkeln, ZÄS 116 (1989), 150; id., MDAIK 48 (1992), 63 (links oben); 64 (Z. 3); $m r$ in Äg. Biogr., 488, c; 510, Z. 4; The Epigraphic Survey, Reliefs III, pl. 22, Z. 2; $W b$ Kairo Nr. 63 (s. $W b$ II, 98, 9).

${ }^{109}$ S. Jansen-Winkeln, $C d E$ 67, 257-8 (6).

${ }^{110} \ddot{A g}$. Biogr., 523, Z. 6; 165 (25). 
Takeloth II. und Osorkon B gebildet hatte. Das ist um so wahrscheinlicher, als von den drei Schenkungsstelen aus der Zeit des Petubastis, die in Unterägypten gefunden wurden, die zwei Exemplare aus Memphis ausgerechnet von Würdenträgern aus Herakleopolis bzw. Pr-Shm-hpr-Re aufgestellt worden sind, ${ }^{111}$ also von Leuten, die zum eigentlichen Machtzentrum Osorkons gehörten und sich möglicherweise seinen Gegnern in Unterägypten angeschlossen hatten. Dies würde auch die für Osorkon offenbar so überraschende und katastrophale Wendung im Jahr 15 Takeloths II. erklären, die ja schwer zu verstehen wäre, wenn es sich nur um eine Erhebung innerhalb seines eigenen Machtbereichs gehandelt hätte.

Es ist interessant, daß auch etwas später, in den auf der Pianchistele geschilderten Vorgängen, eine ganz ähnliche Konstellation erscheint: Dort wird Herakleopolis, das alte militärische Machtzentrum der (22. oder) 23. Dynastie, von Tefnachte aus dem Norden und von Nimlot D, dem Herrscher von Hermopolis, aus dem Süden bedroht und angegriffen. Wenn Petubastis wirklich König von Hermopolis war, wie oben vermutet, hätte sich schon rund $100 \mathrm{Jahr}$ früher etwas ganz Ähnliches ereignet.

Übrigens wäre es nicht ausgeschlossen-zugegeben ein sehr spekulativer Gedankedaß auch Osorkon B, wie später Peftjauawybast, schon Unterstützung aus Nubien bekam. Nach zwei letztlich gescheiterten friedlichen Einigungen (s.o.) verschwindet er nach dem Jahr 29 Schoschenks III., um dann 10 Jahre später als endgültiger Sieger wieder aufzutauchen und schließlich König zu werden. Wenn dieser Sieg schon durch napatanische Unterstützung möglich wurde, wäre dies eine zwanglose Erklärung dafür, daß Oberägypten allmählich unter den Einfluß Nubiens geriet.

\section{Schoschenk II.}

Wie dem auch sei, auf jeden Fall kann Osorkon III. die 'thebanische 23. Dynastie' zunächst stabilisieren, bevor sie von den nubischen Eroberern verdrängt bzw. ersetzt wird. Ob schon Harsiese A zu dieser Dynastie gehört hat oder vielleicht zum rivalisierenden Haus des Petubastis, ist leider gänzlich unklar. Seine bisher angenommene genealogische Verknüpfung beruhte, wie oben gezeigt, jedenfalls auf einem Irrtum. Durch diesen selben Irrtum war auch die Ansicht bedingt, der Hohepriester Schoschenk schreibe seinen Namen auch auf der Besstatue in einer Kartusche. ${ }^{112}$ Tatsächlich tut er dies nur ein einziges Mal, auf der Nilstatue BM EA $8 .{ }^{113}$

Von Schoschenk sind folgende Belege bekannt:

a) Stehfigur des S. Kairo CG $42193 ;{ }^{114}$

b) Stehfigur des S. Kairo CG $42194 ; ;^{115}$

c) Unterteil einer Stehfigur des S. aus dem Luxortempel; ${ }^{116}$

d) von S. gestiftete Nilstatue London BM EA $8 ;{ }^{117}$

${ }^{111} \mathrm{~S} . T I P^{2}$, $§ 300$; für die Behauptung Redfords (King-Lists, 313, n. 113), die beiden Stelen seien erst nachträglich nach Memphis geschafft worden, gibt es keinerlei Anhaltspunkte.

${ }^{112} L R$ III, 331.

${ }^{113}$ S.u., Beleg (d).

${ }^{114} \mathrm{G}$. Legrain, Statues et statuettes de rois et de particuliers, III (Kairo 1914), 3; pl. ii.

${ }^{115}$ Ibid., 3-4; pl. iii-iv.

${ }^{116} \mathrm{PM} \mathrm{II}^{2}, 337$; L. Habachi, 'Clearance of the Area to the East of Luxor Temple and Discovery of Some Objects', $A S A E 51$ (1951), 455-6, pl. iii.

${ }^{117}$ PM II ${ }^{2}, 289$; bester Text bei Lepsuis, Auswahl, pl. xv, a-h. 
e) von S. gestiftete Besstatue Durham (s.o.);

f) Inschrift hinter der westlichen Kolossalstatue am Ausgang des Hofes Ramses' II. im Luxortempel (heute zerstört); ${ }^{118}$

g) Ostraka aus Abydos. ${ }^{119}$

In der Filiation seines Sohnes, des Amunpropheten Osorkon:

h) Papyrus Denon ('B') in St. Petersburg; ${ }^{120}$

i) Papyrus Denon ('C') in St. Petersburg; ${ }^{121}$

j) Sargdeckel Stockholm Medelshavsmuseet NME 838. ${ }^{122}$

Diese Belege enthalten folgende Titel des Schoschenk:

hm-ntr tpj $n$ Fmn-Re njswt ntrw: a; b; d-h;

hm-ntr tpj $n$ Ұmn: i; j;

$m r m s ̌ r ~ w r h ̧ s w t j: \mathrm{a} ; \mathrm{b} ; \mathrm{e}$

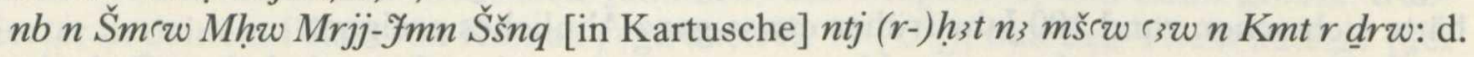

Daraus ergibt sich also folgender Stammbaum: ${ }^{123}$

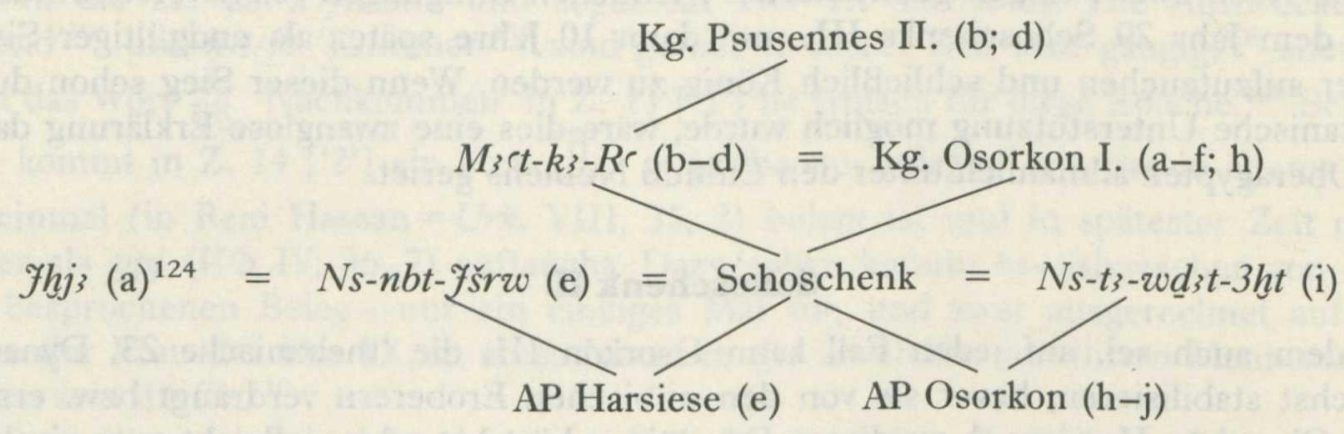

Sollte es sich bei dem im Vorraum des Grabes Psusennes I. in Tanis bestatteten König $H q_{3}$ - $h p r-R e$ Schoschenk um den früheren Hohenpriester Schoschenk handeln, wie oft angenommen wird, ${ }^{125}$ wäre er später König (zumindest als Koregent) in Unterägypten geworden. Sein Name erscheint auf seinem Silbersarg, ${ }^{126}$ der Kartonage, ${ }^{127}$ vier Einge-

${ }^{118}$ Daressy, RecTrav 35, 133; PM II ${ }^{2}, 313$ (71).

${ }^{119} \mathrm{PM} \mathrm{V}, 80$.

${ }^{120}$ V. Denon, Voyage dans la Basse et la Haute Egypte (Paris 1802), pl. 137; J. Lieblein, Die aegyptischen Denkmäler in St. Petersburg, Helsingfors, Upsala und Copenhagen (Christiania 1873), 58; A. Niwiński, Studies on the Illustrated Theban Funerary Papyri (OBO 86, Göttingen 1989), 318-19 ('Leningrad 5').

${ }^{121}$ Denon, Voyage, pl. 138; Lieblein, Denkmäler, 56-7; Niwiński, Studies, 318 ('Leningrad 4').

${ }^{122}$ B. Peterson, 'Ausgewählte ägyptische Personennamen nebst prosopographischen Notizen aus Stockholmer Sammlungen', OrSu 19/20 (1970/71), 17-18.

${ }^{123}$ In Klammer jeweils die Belege; $\mathrm{AP}=$ (einfacher) Amunprophet.

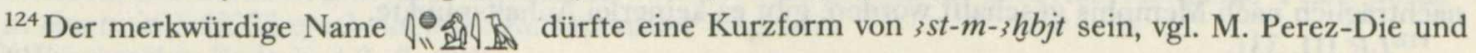

P. Vernus, Excavaciones en Ehnasya el Medina (Madrid 1992), 57 (c).

${ }^{125} T I P^{2}, \S \S 93-4 ; 452$.

${ }^{126}$ P. Montet, La nécropole royale de Tanis, II. Les constructions et le tombeau de Psousennès (Paris 1951), 37-8 u. pl. xvii-xx; Kêmi 9 (1942), 61 u. fig. 44.

${ }^{127}$ Montet, Tanis II, 38-40; Kêmi 9, 62-4; fig. 46-7; pl. xiii; vgl. auch H. Stierlin und Ch. Ziegler, Tanis (Fribourg und Paris 1987), 168 (93). 


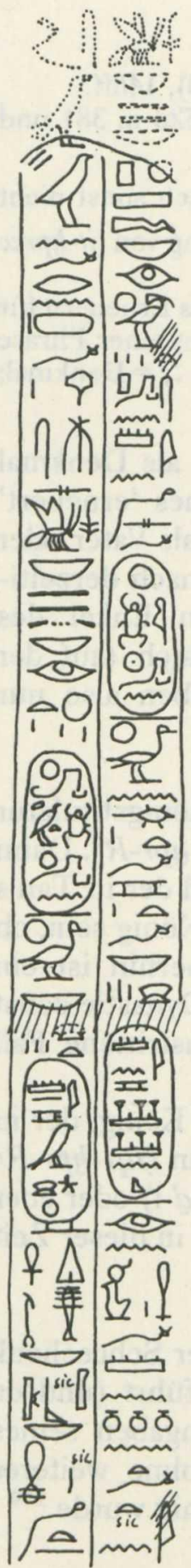

Fig. 5. Kairo CG 42192, Inschrift auf Rückenpfeiler. weidesärgen, ${ }^{128}$ einem Gürtel, ${ }^{129}$ einem Pektoral ${ }^{130}$ und einer Kopfstütze, ${ }^{131}$ und zwar $5 \mathrm{x}$ als Kartuschenpaar Hq3-hpr-Re Stp.n-Re, 16x

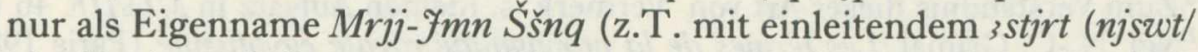
$n b$ Hrw) und $2 \mathrm{x}$ nur als Thronname Hq3-hpr-Re Stp.n-Re (mit einleitendem 3stjrt njswt [nb $\left.t_{3} w j\right]$ ). Gegen eine Identifizierung dieses Schoschenk $H q_{3}-h p r-R r$ in Tanis mit Schoschenk I., wie sie H. Jacquet-Gordon vorgeschlagen hatte, ${ }^{132}$ hat Kitchen ${ }^{133}$ überzeugende Argumente vorgebracht. Es handelt sich also entweder um den Hohenpriester Schoschenk oder um einen sonst unbekannten König.

Damit verknüpft ist der gleichfalls problematische Fall der Statue Kairo CG $42192 .{ }^{134}$ Nach allgemeiner Ansicht ${ }^{135}$ handelt es sich bei dem König Schoschenk, der diese Statue 'erneuert' hat, trotz des divergierenden Thronnamens oß焦 um Schoschenk I., der so seine Verbundenheit mit seinem unmittelbaren Vorgänger aus der 21. Dynastie demonstrierte. In diesem Fall wäre also die Maat-Feder in die 'weiße Krone' zu emendieren. Demgegenüber haben M. Römer ${ }^{136}$ und ich selbst ${ }^{137}$ die Meinung vertreten, daß es sich um Schoschenk II., d.h. den Hohenpriester und Enkel des Psusennes handele. Um diese Ansicht zu untermauern, soll hier einmal die Rückeninschrift von Kairo CG 42192 im Zusammenhang besprochen werden ${ }^{138}$ (s. dazu Fig. 5 und pl. 000):

Z. 1 '(Werk des) ${ }^{a}$ [Königs von Ober- und Unterägypten, des Herrn der Beiden Länder, Herrn] der Kraft, Herrn des Vollbringens von Wohltaten, ${ }^{\mathrm{b}}$ den Amun selbst ausgewählt hat, $M 3$ r-hpr-Re Stp.n-Re, des leiblichen Sohnes des Re, seines geliebten, des Herrn der Kronen $M r j j-\exists m n$ Ššnq, das seine Majestät gemacht hat (als) Denkmal für seinen Vater Amun, ${ }^{\mathrm{c}}$

Z. 2 nachdem er erneuert hat ${ }^{d}$ seinen [des Denkmals] Namen auf den, der ihn erzeugte, den König von Ober- und Unterägypten, den Herrn der Beiden Länder, den Herrn des Rituals Tjt-hprw-Rr Stp.n-Rr, den

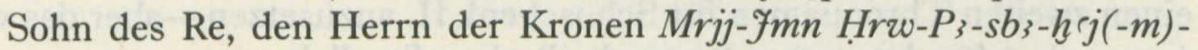
$n w t$, dem alles Leben, Dauer und Herrschaft gegeben ist und alle Gesundheit(?) ewig wie (Re).'

${ }^{128}$ Montet, Tanis II, 56-7 u. pl. xxxv; Kêmi 9, 86-91; pl. xxix-xxx.

${ }^{129}$ Montet, Tanis II, 41-2 u. pl. xxxii; Kêmi 9, 73-4; pl. xxii.

${ }^{130}$ Montet, Tanis II, 42-3 u. pl. xxvi-xxvii; Kêmi 9, 66-8; pl. xx.

${ }^{131}$ Montet, Tanis II, 50 u. pl. xxxiii.

${ }^{132} \mathrm{BiOr} 32,359$; vgl. auch Edwards, $C A H^{2}, \mathrm{III} / 1,549$.

${ }^{133}{ }^{T I P} P^{2}, \S 452$.

${ }^{134}$ Legrain, Statues et statuettes III, 1-2 u. pl. i.

${ }^{135}$ Z. B. LR, III, 316, XLVI; TIP 2 , § 245; Bonhême, Noms royaux, 103; J. Yoyotte,

'A propos de Psousennes II', Bulletin de la Société Française des Fouilles de Tanis,

1 (1988), 41.

136 'Varia zu Psusennes "II" und zur 21. Dynastie', GM 114 (1990), 96.

${ }^{137} \mathrm{BiOr} \mathrm{47,86.}$

${ }^{138} \mathrm{Zu}$ Lesungsverbesserungen gegenüber Legrain s. schon die Kollation von E. Hornung und E. Staehelin bei R. Giveon in Fragen an die altägyptische Literatur (Fs. Otto), hrsg. J. Assmann et al. (Wiesbanden 1977), 174-5, n. 4. 


\section{Anmerkungen}

a Zum Verständnis dieser Art von 'Vermerk' s. meinen Aufsatz in MDAIK 46 (1990), $146 \mathrm{ff}$.

${ }^{b}$ Vgl. N.-C. Grimal, Les termes de la propagande royale égyptienne (Paris 1986), 523 n. 381 und 513 n. 346. Die Ergänzung $[t]$ bei $3 h w(t)$ ist fraglich.

c. a kann wohl nur ein Fehler für $7 m n$ sein. Man beachte, daß die Inschrift auch sonst nicht fehlerfrei ist: Vor allem das Ende von Z. 2 ist sehr eigenartig, ebenso die Schreibung von tjt-hprw im Thronnamen des Psusennes.

${ }^{d}$ Möglich und phraseologisch sogar naheliegender wäre der Infinitiv: '(nämlich) das Erneuern für ihn [Amun]...' Er ist allerdings deshalb weniger wahrscheinlich, weil man dann in einer Phrase gleich drei maskuline Pronomina mit unterschiedlichem Bezug hätte $(. f=$ Amun; $f=$ Denkmal; $s w=$ Schoschenk).

Die Statue ist also einerseits von einem König Schoschenk dem Amun als Denkmal (neu) geweiht worden, zum anderen ist sie auf den Namen des Psusennes 'erneuert' worden, und dieser Psusennes wird unmißverständlich als Erzeuger, d.h. als Vater oder doch Vorvater dieses Schoschenk bezeichnet. Demzufolge kann es sich-nach derzeitiger Kenntnis-nur um den Hohenpriester Schoschenk handeln, den Enkel des Psusennes, auf keinen Fall aber um Schoschenk I., dessen Vorfahren sich (auf der Pasenhorstele) über viele Generationen zurückverfolgen lassen. Es bleiben also nur folgende Möglichkeiten:

1. Es handelt sich um den Hohenpriester Schoschenk, der hier die volle Königstitulatur angenommen hat, aber nicht um den in Tanis bestatteten Schoschenk $H q_{3}-h p r-R$ r. Dann lautet der Thronname dieses 'neuen' thebanischen Pharao Msr-hpr-Rr, und der in Tanis bestattete Schoschenk müßte ein aus anderen Quellen völlig unbekannter König sein, ob er nun eine eigene Regierungszeit hatte oder nur Koregent war. Immerhin ist ein thebanischer Hoherpriester, der später König in Tanis wurde, in der 22. Dynastie sonst nicht bezeugt, und auch in der 21. käme nur der sehr dunkle und umstrittene Fall Psusennes II./III. in Frage.

2. Es handelt sich um den Hohenpriester Schoschenk und um denselben König, der in Tanis als $H q_{3}-h p r-R e$ begraben ist. Dann hätte man entweder $M 3 r-h p r-R r$ in $H_{i} q_{3}-h p r-R r$ zu verbessern (auf jeden Fall eine geringfügigere Emendation als $m_{3}$ ? zu $h \underline{d} \underline{d}$ !) oder aber einen zweiten Thronnamen für Schoschenk II. anzusetzen-aber das wäre in dieser Zeit (zumindest für den ersten Bestandteil) ohne Parallele.

Irritierend an beiden Möglichkeiten ist die Tatsache, daß der Hohepriester Schoschenk auf zeitgenössischen Denkmälern nur ein einziges Mal den Königstitel führt (und da auch nur den Eigennamen in Kartusche) und sogar in den Filiationsangaben seines Sohnes Osorkon immer nur Hoherpriester ist. Aber es ist natürlich ohne weiteres möglich, daß diese Denkmäler alle entstanden sind, bevor Schoschenk König wurde. ${ }^{139}$

\footnotetext{
${ }^{139}$ Nach Einreichen des Manuskripts dieses Aufsatzes ist die Inschrift CG 42192 auch von J. von Beckerath neu veröffentlicht worden ('Zur Rückeninschrift der Statuette Kairo CG 42192', Or 63 (1994), 84-7). Auch er ist jetzt der Meinung, daß der Hohepriester Schoschenk II. ihr Stifter ist (ebenso N. Dautzenberg, $G M 144,21)$. In einem Nachtrag $(\operatorname{Or} 63,87)$ schlägt er vor, daß Schoschenk, $H q_{3}$ - $h p r-R r$ aus Tanis eine von dem Hohenpriester Schoschenk (M $\left.M_{3} r-h p r-R r\right)$ verschiedene Person sei, vermutlich ein Sohn Takeloths I. und ephemerer Vorgänger Osorkons II. (ebenso in GM 144, 8-9). Auch hier ist Dautzenberg unabhängig davon zur selben Ansicht gelangt (GM 144, 21-4).
} 
ТАв. 1. Kondordanz und Belegstellen zur 'oberägyptischen 23. Dynastie'

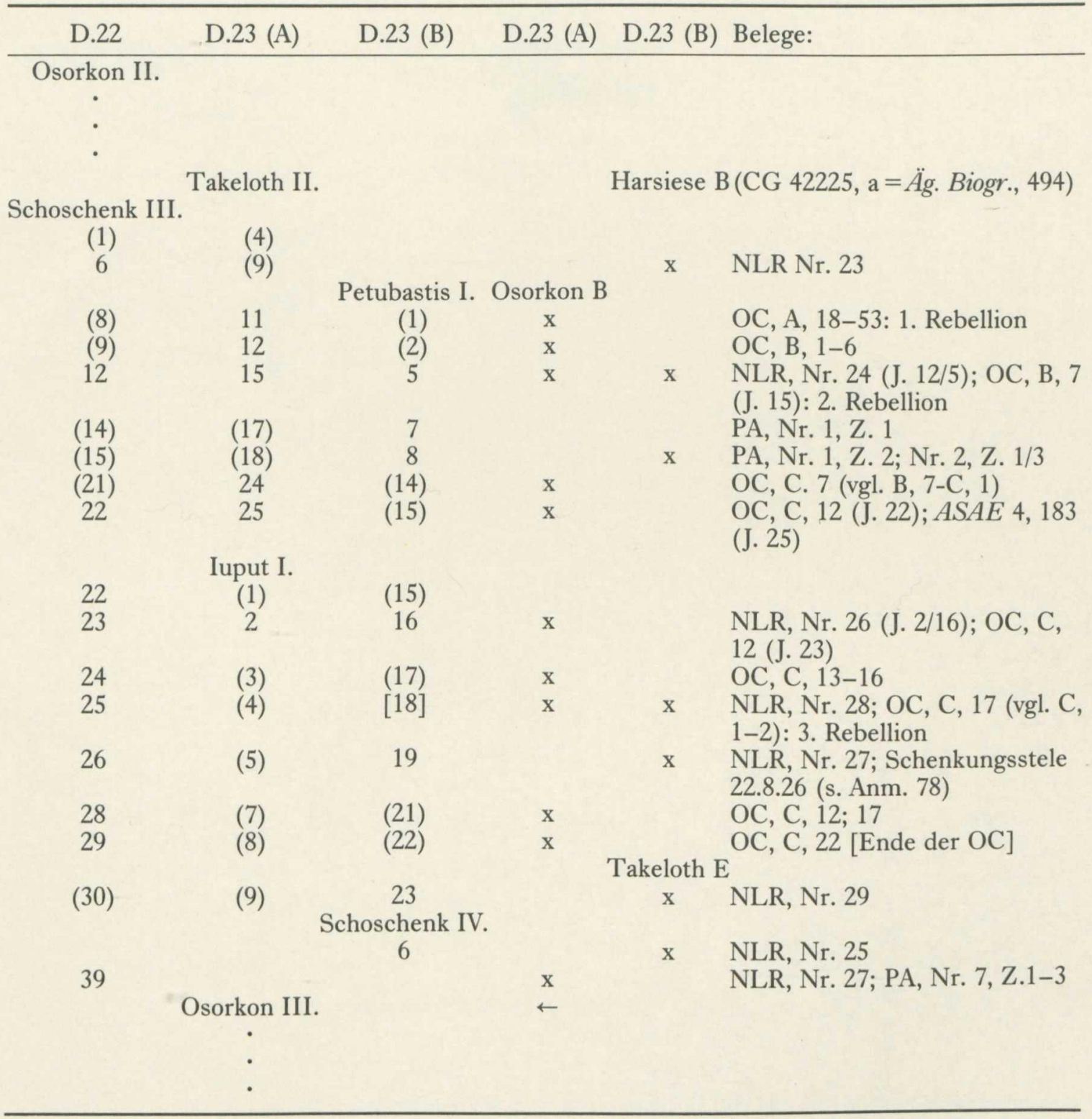

$\mathrm{NLR}=$ Nile Level Records, s. von Beckerath, $¥ A R C E$ 5, 43-55; PA= Priesterannalen, s. Legrain, RecTrav 22, 51-63; Daressy, RecTrav 35, 131-2; Kruchten, Les annales (s.o., n. 254); $\mathrm{OC}=$ Osorkonchronik (die Bezeichnung der verschiedenen Teile der Inschrift durch A, B und C nach CPO: $\mathrm{A}=$ The Epigraphic Survey, Reliefs III, pl. 16-19; $\mathrm{B}=$ ibid., pl. 21; $\mathrm{C}=$ ibid., pl. 22). 

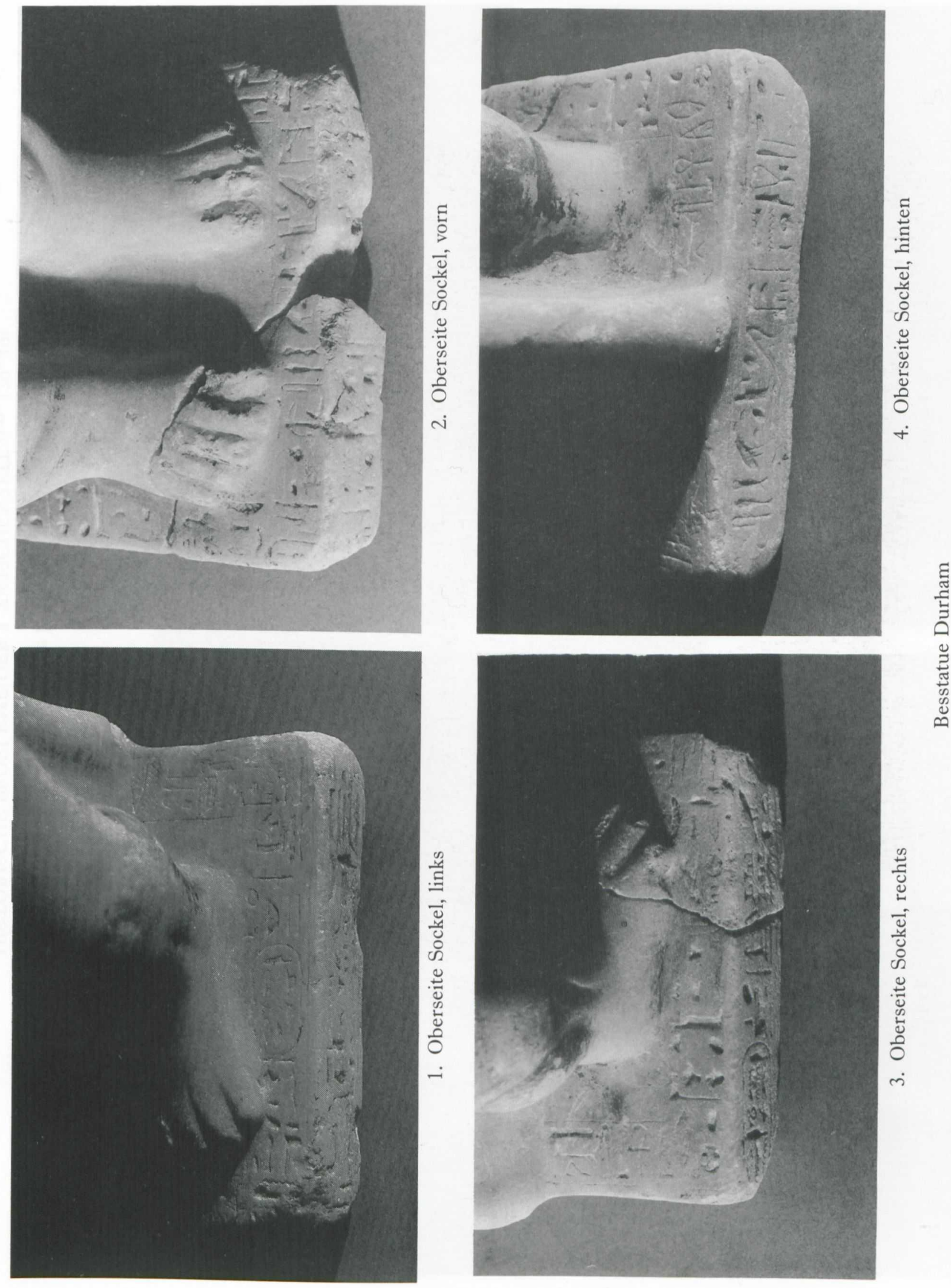

京 

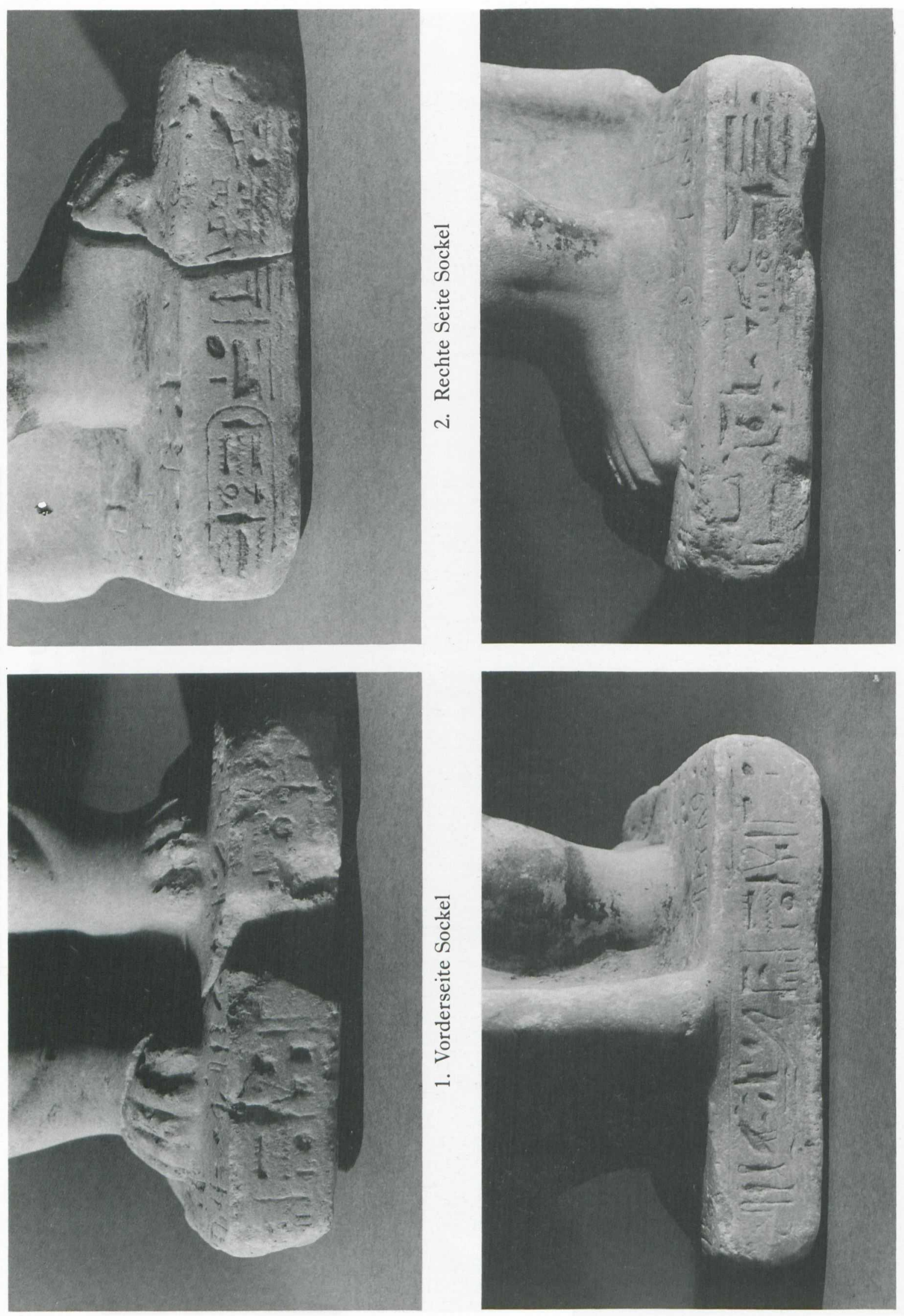

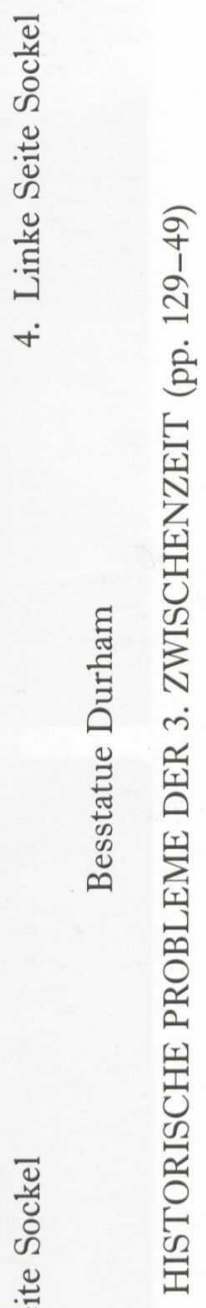



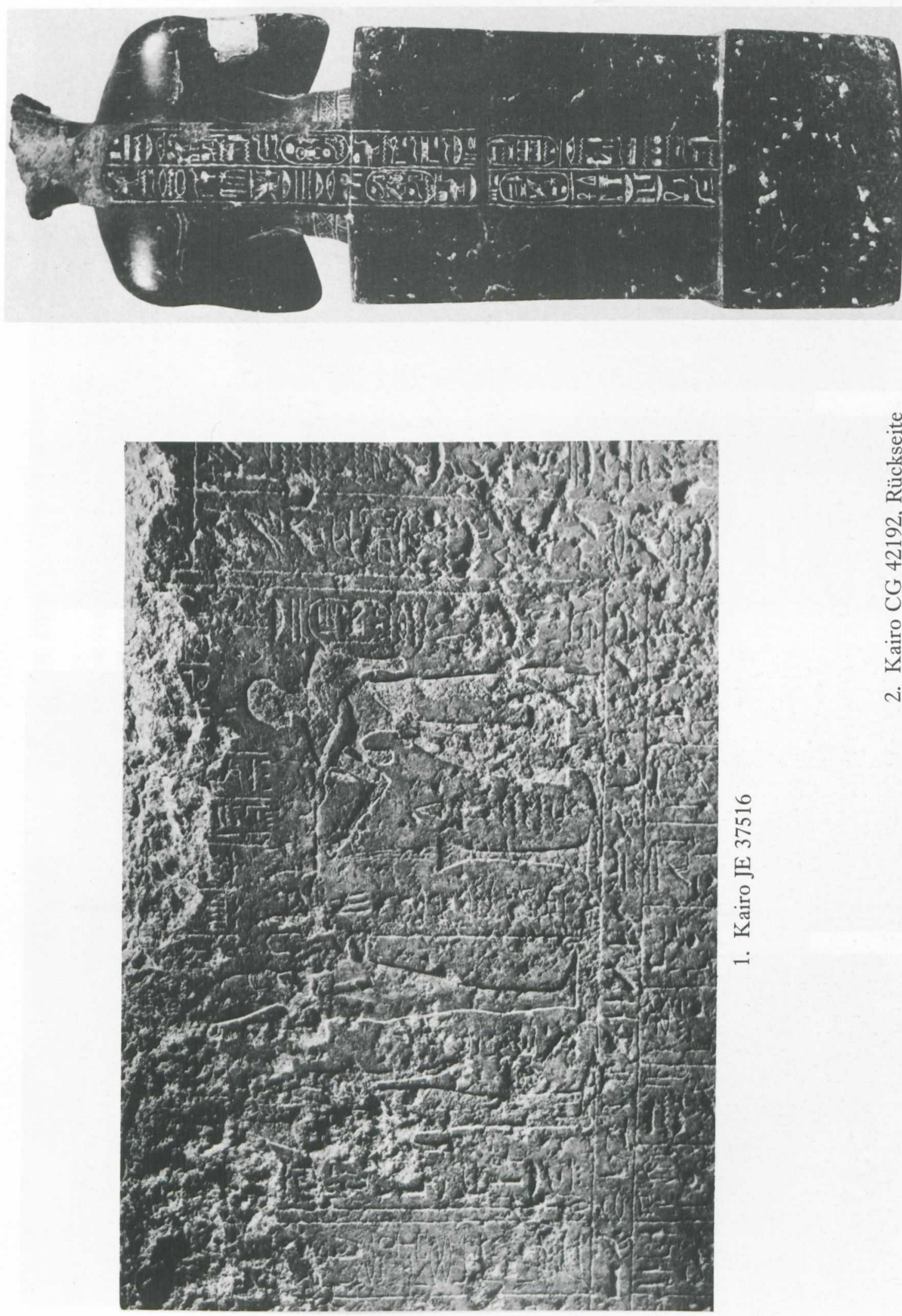